NBER WORKING PAPER SERIES

OPTIMAL TAXATION IN AN RBC MODEL:
A LINEAR-QUADRATIC APPROACH

\author{
Pierpaolo Benigno \\ Michael Woodford \\ Working Paper 11029 \\ http://www.nber.org/papers/w11029
}

\author{
NATIONAL BUREAU OF ECONOMIC RESEARCH \\ 1050 Massachusetts Avenue \\ Cambridge, MA 02138 \\ January 2005
}

Presented by the second author as a Plenary Lecture at the 10th Annual Conference on Computing in Economics and Finance, Amsterdam, Netherlands, July 8-10, 2004. We thank Vasco Curdia and Mauro Roca for excellent research assistance, Ken Judd, Jinill Kim, Andy Levin and Willi Semmler for helpful comments, and the National Science Foundation for research suppport. The views expressed herein are those of the author(s) and do not necessarily reflect the views of the National Bureau of Economic Research.

(C) 2005 by Pierpaolo Benigno and Michael Woodford. All rights reserved. Short sections of text, not to exceed two paragraphs, may be quoted without explicit permission provided that full credit, including $\odot$ notice, is given to the source. 
Optimal Taxation in an RBC Model: A Linear-Quadratic Approach

Pierpaolo Benigno and Michael Woodford

NBER Working Paper No. 11029

January 2005

JEL No. C63, H21

\title{
ABSTRACT
}

We reconsider the optimal taxation of income from labor and capital in the stochastic growth model analyzed by Chari et al. (1994, 1995), but using a linear-quadratic (LQ) approximation to derive a log-linear approximation to the optimal policy rules. The example illustrates how inaccurate "naive" LQ approximation --- in which the quadratic objective is obtained from a simple Taylor expansion of the utility function of the representative household --- can be, but also shows how a correct LQ approximation can be obtained, which will provide a correct local approximation to the optimal policy rules in the case of small enough shocks. We also consider the numerical accuracy of the LQ approximation in the case of shocks of the size assumed in the calibration of Chari et al. We find that the correct LQ approximation yields results that are quite accurate, and similar in most respects to the results obtained by Chari et al. using a more computationally intensive numerical method.

\author{
Pierpaolo Benigno \\ Department of Economics \\ New York University \\ 269 Mercer Street \\ New York, NY 10003 \\ pierpaolo.benigno@nyu.edu \\ Michael Woodford \\ Department of Economics \\ Columbia University \\ $420 \mathrm{~W} .118^{\text {th }}$ Street \\ New York, NY 10027 \\ and NBER \\ mw2230@columbia.edu
}


Linear-quadratic (LQ) optimal-control problems have been the subject of an extensive literature. ${ }^{1}$ It is not clear, however, how likely it is that optimal policy problems with explicit microfoundations - that is, policy problems in which both the assumed objective of policy and the constraints on possible outcomes are derived from an explicit account of the decision problems of private agents - should take this form. Elsewhere (Benigno and Woodford, 2004b), we show that it is possible in a broad class of models to derive an LQ problem that locally approximates an exact policy problem, in the sense that the solution to the LQ problem represents a local linear approximation to the solution to the exact problem, that will describe it with arbitrary accuracy in the case of small enough random disturbances. It does not generally suffice for this purpose to define an LQ problem in which the objective is a local quadratic approximation to the exact objective and the constraints are local linear approximations to the exact constraints. ${ }^{2}$ Nonetheless, we show that it is quite generally possible to derive a correct LQ approximation, if sufficient care is taken in the choice of the quadratic objective.

Here we illustrate both the potential problems with naive LQ approximation and the application of our own method in the context of a well-known example, the analysis of dynamic optimal taxation of income from labor and capital in an RBC model, treated by Chari et al. (1994). The example is of interest not only because it is a simple case in which naive LQ approximation would lead to extremely incorrect conclusions, but also because the paper of Chari et al. is often cited as evidence that log-linearization is dangerous in the context of optimal tax policy problems, even if it can be used with fair accuracy in other contexts (such as the approximate characterization of the aggregate fluctuations implied by an RBC model).

In fact, Chari et al. use a minimum-weighted-residual method that is computationally more difficult than ours to numerically characterize the optimal dynamics of capital and labor taxes, and state that they do so because a log-linear approximation was found to be quite inaccurate. ${ }^{3}$ We therefore consider the accuracy of the local linear approximation provided by our LQ approach in the case of disturbances of the amplitude assumed by Chari et al. in their numerical work. We do this both

\footnotetext{
${ }^{1}$ Important references include Bertsekas (1976), Chow (1975), Hansen and Sargent (2004), Kwakernaak and Sivan (1972), and Sargent (1987).

${ }^{2}$ The problem with "naive" LQ approximation of this sort is discussed, for example, by Judd (1996, sec. 4; 1999, pp. 505-508).

${ }^{3}$ They provide further details of the nature of the supposed inaccuracy of log-linearization in Chari et al. (1995).
} 
by comparing our results with those obtained by Chari et al. using their preferred method, and also by comparing them with those that would be obtained through a second-order perturbation analysis of the exact conditions characterizing optimal policy. We find that the second-order perturbation solution (a local approximation that is accurate to second order in the amplitude of the disturbances, rather than only to first order) differs only slightly from the linear approximation provided by our LQ approach. Similarly, the numerical results of Chari et al. are much closer to those implied by our log-linear approximation to optimal policy than their discussion of the accuracy of log-linearization suggests. Hence we find that an LQ approximation, when carried out correctly, gives a useful account of the way in which capital and labor taxes should optimally be adjusted in response to real disturbances.

In section 1, we recapitulate the analysis of optimal tax policy in a real business cycle model of Chari et al., in order to be clear about the policy problem that we wish to approximate. In section 2, we first show how naive LQ approximation of this problem would lead to incorrect conclusions, and then show how a correct LQ approximation can instead be derived. In section 3, we calibrate the model in accordance with the assumptions made in the analysis of Chari et al. (1994, 1995), and use our LQ approach to compute some of the statistics regarding the optimal dynamics of capital and labor taxes that they report; we then compare our results both with theirs and with those obtained using a second-order perturbation technique. Section 4 concludes.

\section{The Optimal Policy Problem}

We begin by recalling the optimal policy problem analyzed by Chari et al. We recall this, not only in order to be clear about the problem for which we seek to derive an approximate solution, but also because our definition of the optimal policy problem differs slightly from the presentation in the papers of Chari et al. Like these authors, we are only interested in characterizing the stationary fluctuations in the capital and labor tax rates that occur asymptotically under a Ramsey-optimal policy. However, the stationary fluctuations that occur asymptotically under the (unconstrained) Ramsey policy represent the solution to a constrained version of the usual Ramsey problem (what we call optimal policy "from a timeless perspective"), and it is this constrained problem that we wish to approximate by an appropriately 
defined LQ problem.

\subsection{The Model}

As in Chari et al. (1994), we extend a standard RBC model to include proportional tax rates on labor and capital income, and the possibility for the government to issue non-state-contingent real debt. ${ }^{4}$ There is a continuum of measure one of households (here indexed by $j$ ) with identical time-separable preferences

$$
U_{t}^{j}=E_{t} \sum_{T=t}^{\infty} \beta^{T-t} u\left(c_{T}^{j}, h_{T}^{j}\right),
$$

with $0<\beta<1$. The period utility function $u$ is strictly increasing and concave in consumption, $c$, and in the negative of hours worked, $-h$, and is continuously differentiable and satisfies standard Inada conditions.

In addition to holding capital, households can also invest in a set of state-contingent one-period real securities that span all of the states of nature that the households may face in the next period. These securities are in zero net supply, except for the riskless debt that is issued by the government. Each household is subject to a flow budget constraint of the form

$$
c_{t}^{j}+\left(k_{t+1}^{j}-k_{t}^{j}\right)+b_{t}^{j} \leq a_{t}^{j}+\left(1-\tau_{t}^{k}\right)\left(\rho_{t}-\delta\right) k_{t}^{j}+\left(1-\tau_{t}^{h}\right) w_{t} h_{t}^{j},
$$

where $k_{t}^{j}$ the stock of capital goods that it owns and rents to firms in period $t$, and $0 \leq \delta \leq 1$ is the depreciation rate. In the budget constraint (1.2), $b_{t}^{j}$ denotes the household j's value of the period-t portfolio of contingent securities which delivers a state-contingent return $a_{t+1}$ at time $t+1$. The complete-markets assumption implies the existence of a unique stochastic discount factor $r_{t, t+1}$ such that

$$
b_{t}^{j}=E_{t}\left[r_{t, t+1} a_{t+1}^{j}\right]
$$

\footnotetext{
${ }^{4}$ Chari et al. consider a more general framework, with a broader set of securities that may be issued by the government. But they show that this does not increase the set of equilibrium allocations that can be achieved through an appropriate policy, and that the presence of redundant policy instruments simply results in indeterminacy of certain aspects of optimal policy. Here we simplify the analysis, and obtain determinate results regarding the optimal state-contingent tax rate on income from capital, by assuming only two dimensions along which policy can be varied each period.
} 
which can be substituted into (1.2) to yield

$$
c_{t}^{j}+\left(k_{t+1}^{j}-k_{t}^{j}\right)+E_{t}\left[r_{t, t+1} a_{t+1}^{j}\right] \leq a_{t}^{j}+\left(1-\tau_{t}^{k}\right)\left(\rho_{t}-\delta\right) k_{t}^{j}+\left(1-\tau_{t}^{h}\right) w_{t} h_{t}^{j} .
$$

Households can rent their capital goods to firms at an economy-wide rental rate, given by $\rho_{t}$, and they work for an as well economy-wide wage rate given by $w_{t}$. The returns to these services are taxed at the rates $\tau_{t}^{k}$ and $\tau_{t}^{h}$, respectively. Finally, the household is subject to an appropriate set of borrowing limits.

The household's optimization problem involves maximizing the utility function (1.1) under the flow budget constraints (1.4) and the borrowing limits, given the initial condition $k_{t_{0}} \geq 0$, subject to the additional constraints that $c_{t}^{j} \geq 0, h_{t}^{j} \geq$ $0, k_{t+1}^{j} \geq 0$ for each $t \geq t_{0}$. The Inada conditions on the utility function ensure that corner solutions can be ignored. Necessary and sufficient conditions for household optimization are then:

1. The first-order conditions (FOCs) for the optimal allocation of consumption spending are

$$
u_{c}\left(c_{t}^{j}, h_{t}^{j}\right) r_{t, t+1}=\beta u_{c}\left(c_{t+1}^{j}, h_{t+1}^{j}\right),
$$

for each time $t$ and each contingency at time $t+1$. Conditions (1.5) imply the stochastic Euler equation

$$
u_{c}\left(c_{t}^{j}, h_{t}^{j}\right)=\beta\left(1+r_{t}\right) E_{t} u_{c}\left(c_{t+1}^{j}, h_{t+1}^{j}\right),
$$

where $r_{t}$ is the risk-free one-period real rate defined by

$$
1+r_{t} \equiv\left[E_{t} r_{t, t+1}\right]^{-1}
$$

2. The FOCs for optimal capital accumulation are

$$
u_{c}\left(c_{t}^{j}, h_{t}^{j}\right)=\beta E_{t}\left\{u_{c}\left(c_{t+1}^{j}, h_{t+1}^{j}\right)\left[\left(1-\tau_{t+1}^{k}\right)\left(\rho_{t+1}-\delta\right)+1\right]\right\}
$$

for each date $t$.

3. The FOCs for optimal labor supply are

$$
\frac{u_{h}\left(c_{t}^{j}, h_{t}^{j}\right)}{u_{c}\left(c_{t}^{j}, h_{t}^{j}\right)}=-\left(1-\tau_{t}^{h}\right) w_{t}
$$

for each date $t$. 
4. Finally, it is necessary that the household exhaust its intertemporal budget constraint.

There is similarly a continuum of measure one of firms, which each produce a homogeneous good using the same technology in competitive product and factor markets. The good can be purchased by households, and used for both consumption and investment (capital accumulation) purposes, and can also be consumed by the government. Each firm's production technology is of the form $y_{t}^{i}=f\left(k_{t}^{i}, z_{t} h_{t}^{i}\right)$ where $f$ is a constant-returns-to-scale function and $z$ is an exogenous labor-augmenting technology shock. Firms maximize their profits

$$
\pi_{t}^{i}=f\left(k_{t}^{i}, z_{t} h_{t}^{i}\right)-\rho_{t} k_{t}^{i}-w_{t} h_{t}^{i}
$$

First-order necessary and sufficient conditions for this imply that

$$
f_{k}\left(k_{t}^{i}, z_{t} h_{t}^{i}\right)=\rho_{t}
$$

and

$$
z_{t} f_{h}\left(k_{t}^{i}, z_{t} h_{t}^{i}\right)=w_{t}
$$

at each time $t$. Given the symmetric structure of the model, it is clear that all households make the same optimal choices at each date, and similarly for all firms; we can thus omit the superscripts $i$ and $j$ in what follows.

It remains to describe the income and expenditure of the government. The government purchases goods and raises revenues through taxes on the services of capital and labor. We assume that the government can borrow by issuing a one-period riskfree real bond. The real value of government debt evolves according to the law of motion

$$
b_{t}^{g}=b_{t-1}^{g}\left(1+r_{t-1}\right)-s_{t}
$$

where $b_{t}^{g}$ denotes the end-of-period liabilities of the government in units of these oneperiod bonds, $r_{t}$ is the one-period real interest rate between periods $t$ and $t+1$, and $s_{t}$ is the real primary government budget surplus. The latter quantity is defined by

$$
s_{t} \equiv \tau_{t}^{k}\left(\rho_{t}-\delta\right) k_{t}+\tau_{t}^{h} w_{t} h_{t}-g_{t},
$$

where $g_{t}$ denotes government purchases of the good. Government purchases are treated as an exogenously given stochastic process, rather than a policy decision 
analyzed here. Rational-expectations equilibrium requires that the expected path of government surpluses must satisfy an intertemporal solvency condition

$$
b_{t-1}^{s}=E_{t} \sum_{T=t}^{\infty} r_{t, T}\left[\tau_{T}^{k}\left(\rho_{T}-\delta\right) k_{T}+\tau_{T}^{h} w_{T} h_{T}-g_{T}\right]
$$

in each state of the world that may be realized at date $t$, where $b_{t-1}^{s} \equiv b_{t-1}^{g}\left(1+r_{t-1}\right)$ is the value owed by the government at the beginning of period $t$, i.e., the value at maturity of the debt issued in period $t-1$. (Because the debt is riskless, the value of this variable is known at date $t-1$.)

Finally, goods market equilibrium requires that

$$
y_{t}=f\left(k_{t}, z_{t} h_{t}\right)=c_{t}+g_{t}+k_{t+1}-(1-\delta) k_{t} .
$$

\subsection{Optimal Policy}

In a standard Ramsey policy problem, ${ }^{5}$ the government chooses state-contingent paths for $\left\{\tau_{t}^{k}, \tau_{t}^{h}, b_{t}^{s}, c_{t}, h_{t}, r_{t, t+1}, k_{t+1}, w_{t}, \rho_{t}\right\}$ for all periods $t \geq t_{0}$ that satisfy conditions (1.5), (1.7), (1.8), (1.10), (1.11), (1.14), (1.15) at each time $t$, given initial conditions $b_{t_{0}-1}^{s}$ and $k_{t_{0}}$. We first write this problem in the more compact form presented by Chari et al., and then discuss the closely related problem that we actually approximate.

We first note that we can use condition (1.5) to substitute for $r_{t, T}$ in (1.14), yielding

$$
V_{t}=E_{t} \sum_{T=t}^{\infty} \beta^{T-t} u_{c}\left(c_{T}, h_{T}\right)\left[f\left(k_{T}, z_{T} h_{T}\right)-\left(1-\tau_{T}^{k}\right)\left(\rho_{T}-\delta\right) k_{T}-\delta k_{T}-\left(1-\tau_{T}^{h}\right) w_{T} h_{T}-g_{T}\right]
$$

where we define

$$
V_{t} \equiv b_{t-1}^{s} u_{c}\left(c_{t}, h_{t}\right)
$$

and make use of the relation

$$
f\left(k_{t}, z_{t} h_{t}\right)=\rho_{t} k_{t}+w_{t} h_{t} .
$$

\footnotetext{
${ }^{5}$ Note that this is not precisely the problem considered by Chari et al., for reasons discussed below.
} 
We can furthermore substitute (1.8) into (1.16) for the labor tax rate, and similarly use (1.7) to replace the expected future capital tax rate terms, yielding

$$
W_{t}=b_{t-1}^{s} u_{c}\left(c_{t}, h_{t}\right)+u_{c}\left(c_{t}, h_{t}\right) k_{t}\left[1+\left(1-\tau_{t}^{k}\right)\left(f_{k}\left(k_{t}, z_{t} h_{t}\right)-\delta\right)\right],
$$

where we have also used (1.10) to substitute for $\rho_{t}$, and we define

$$
W_{t} \equiv E_{t} \sum_{T=t}^{\infty} \beta^{T-t}\left[u_{c}\left(c_{T}, h_{T}\right) c_{T}+u_{h}\left(c_{T}, h_{T}\right) h_{T}\right] .
$$

Thus the Ramsey policy problem can equivalently be stated as one of choosing statecontingent paths of $\left\{\tau_{t}^{k}, b_{t}^{s}, c_{t}, h_{t}, k_{t+1}\right\}$ for each $t \geq t_{0}$ to maximize the utility (1.1), subject to the constraints that (1.15), (1.18), (1.19) and

$$
u_{c}\left(c_{t}, h_{t}\right)=\beta E_{t}\left\{u_{c}\left(c_{t+1}, h_{t+1}\right)\left[1+\left(1-\tau_{t+1}^{k}\right)\left(f_{k}\left(k_{t+1}, z_{t+1} h_{t+1}\right)-\delta\right)\right]\right\}
$$

hold at each date $t \geq t_{0}$, given the initial conditions $b_{t_{0}-1}^{s}$ and $k_{t_{0}}$.

In the case that there is no limit on the size of the taxes that may be levied ex post on existing capital, this problem is equivalent to one of choosing the sequences $\left\{c_{t}, h_{t}, k_{t+1}\right\}_{t=t_{0}}^{\infty}$ to maximize (1.1) subject only to the constraint (1.15) for each $t \geq t_{0}$, given the initial condition $k_{t_{0}}$. For one can show that in the case of any sequences $\left\{c_{t}, h_{t}, k_{t+1}\right\}$ satisfying (1.15) given the initial capital stock, and any initial public debt $b_{t_{0}-1}^{s}$, it is possible to construct sequences $\left\{W_{t}, b_{t}^{s}, \tau_{t}^{k}\right\}$ that satisfy the other constraints as well. Note that (1.18) together with (1.20) implies that

$$
u_{c}\left(c_{t}, h_{t}\right) k_{t+1}=\beta E_{t}\left[W_{t+1}-b_{t}^{s} u_{c}\left(c_{t+1}, h_{t+1}\right)\right] .
$$

Then given sequences $\left\{c_{t}, h_{t}, k_{t+1}\right\}$ satisfying (1.15), one can solve (1.19) for the implied sequence $\left\{W_{t}\right\}_{t=t_{0}}^{\infty}$, then solve (1.21) for the implied sequence $\left\{b_{t}^{s}\right\}_{t=t_{0}}^{\infty}$, and finally solve (1.18) for the implied sequence $\left\{\tau_{t}^{k}\right\}_{t=t_{0}}^{\infty}$. The constructed sequences necessarily satisfy conditions (1.18), (1.19), and (1.21) each period in addition to (1.15), and as a consequence they satisfy (1.20) each period as well.

It then follows that the Ramsey policy achieves the same (fully efficient) allocation of resources, regardless of the size of the initial public debt $b_{t_{0}}^{s}$; an initial levy on the pre-existing capital stock is simply used to raise whatever amount of government revenue is needed in the initial period to make it possible to pay off this debt and also finance all subsequent government purchases without any need for distorting 
taxes. ${ }^{6}$ The possibility of doing this, of course, depends in general on the possibility of choosing a large value for $\tau_{t_{0}}^{k}$, possibly one much larger than 1 . This is not especially realistic, and such an assumption makes the problem of optimal taxation too trivial. Consequently, Chari et al., like many other authors, assume a limit on the degree to which it is possible for the government to tax initially existing assets; specifically, they assume that the initial tax rate on capital income $\left(\tau_{t_{0}}^{k}\right)$ is given by a prior commitment, though it may be freely chosen in all later periods.

We assume a constraint on initial policy in the same spirit, but specified slightly differently, so as to make the policy problem recursive (unlike the precise problem defined by Chari et al.); this has the technical advantage of making the optimal policy consistent with a steady state, in the case of an appropriate initial capital stock and in the absence of random disturbances, so that we can then approximate optimal policy in the case of small enough disturbances using Taylor expansions of our objective and constraints around this steady state. ${ }^{7}$ Specifically, we consider the problem of choosing state-contingent paths of $\left\{\tau_{t}^{k}, b_{t}^{s}, c_{t}, h_{t}, k_{t+1}\right\}$ for each $t \geq t_{0}$ to maximize the utility (1.1), subject to the constraints that (1.15), (1.18), (1.19) and (1.20) hold at each date $t \geq t_{0}$, given the initial conditions $b_{t_{0}-1}^{s}$ and $k_{t_{0}}$, and such that in addition a constraint of the form

$$
W_{t_{0}}=\bar{W}_{t_{0}}
$$

is satisfied, where $W_{t_{0}}$ is defined by (1.19), and $\bar{W}_{t_{0}}$ is a pre-existing state-contingent commitment regarding the value (in marginal utility units) of the assets (debt and capital) with which the representative household begins period $t_{0}$. (The latter interpretation of the commitment is seen from (1.18).) This commitment obviously implies a limit on the extent to which tax revenues can be raised by taxing initially existing capital, though there is no limit on the extent to which one may plan to tax capital in later periods.

Following exactly the same argument as above, one can show that this constrained problem is equivalent to a simpler problem, namely, choosing sequences $\left\{W_{t}, c_{t}, h_{t}, k_{t+1}\right\}_{t=t_{0}}^{\infty}$ to maximize (1.1) subject only to the constraint that (1.15) hold

\footnotetext{
${ }^{6}$ In our version of the model, unanticipated ex post variation in the tax rate on capital would still be used as a substitute for state-contingent public debt, in order to ensure intertemporal government solvency in all states of the world.

${ }^{7}$ In Benigno and Woodford (2004b), we discuss recursive policy problems of this kind more generally.
} 
for each $t \geq t_{0}$, given the initial condition $k_{t_{0}}$, and to constraint (1.22), where $W_{t_{0}}$ is defined by (1.19). Once again, the optimal allocation (and the level of household utility obtained) is independent of the initial condition $b_{t_{0}-1}^{s}$, as variations in the initial level of public debt can still be completely offset by variations in the size of the levy on initial capital, though the value of total initial household wealth (in marginalutility units) is constrained to equal $\bar{W}_{t_{0}}$. The value function for this problem can therefore be written as $J\left(k_{t_{0}}, \bar{W}_{t_{0}} ; \xi_{t_{0}}\right)$, where we use the notation $\xi_{t}$ to refer to the exogenous state of the world at date $t$ (including all information available at $t$ about the probability of various exogenous disturbances at any later dates). ${ }^{8}$

This constrained policy problem is recursive, in the following sense. It can be shown to be equivalent ${ }^{9}$ to solving a sequence of policy problems, at each date $t \geq$ $t_{0}$, where the policy problem at date $t$ is to choose values $\left(c_{t}, h_{t}, k_{t+1}\right)$, and statecontingent commitments $\bar{W}_{t+1}$ for each possible state of the world in the following period, so as to maximize

$$
u\left(c_{t}, h_{t}\right)+\beta E_{t} J\left(k_{t+1}, \bar{W}_{t+1} ; \xi_{t+1}\right),
$$

subject to the constraints (1.15) and

$$
\bar{W}_{t}=u_{c}\left(c_{t}, h_{t}\right) c_{t}+u_{h}\left(c_{t}, h_{t}\right) h_{t}+\beta E_{t} \bar{W}_{t+1}
$$

given the values for $k_{t}$ and $\bar{W}_{t}$ determined in the previous period. Here $J(k, \bar{W} ; \xi)$ is the value function for the problem defined in the previous paragraph; it can be shown that the value of the single-period decision problem just defined is also given by $J\left(k_{t}, \bar{W}_{t} ; \xi_{t}\right)$. Given the state-contingent paths $\left\{W_{t}, c_{t}, h_{t}, k_{t+1}\right\}$ that solve this problem, the associated paths for the public debt and tax rates are given by (1.8), (1.18), (1.21), and the initial condition $b_{t_{0}-1}^{s}$.

As noted above, Chari et al. assume a somewhat different constraint on policy in period $t_{0}$. However, one can show that the policy problem that they define is equivalent to a two-stage problem, in which values $\left(c_{t_{0}}, h_{t_{0}}, k_{t_{0}+1}\right)$ and state-contingent commitments $\bar{W}_{t_{0}+1}$ are chosen in the first stage, subject to the initial constraint specified by Chari et al. (rather than a constraint on the value of $W_{t_{0}}$ ), and policy

\footnotetext{
${ }^{8}$ This corresponds simply to the vector of exogenous disturbances at date $t$ if each disturbance is Markovian, as in the quantitative example treated below.

${ }^{9}$ The proof follows the same lines as the proof of Proposition 2 in Benigno and Woodford (2004a), and is omitted here.
} 
from date $t_{0}+1$ onward is chosen to solve the kind of constrained problem defined here, given the commitments $\bar{W}_{t_{0}+1}$ chosen in the first stage. ${ }^{10}$ Hence if the optimal dynamics of tax rates and other endogenous variables eventually exhibit stationary fluctuations (as they find to be the case), these stationary fluctuations correspond to the equilibrium dynamics under a constrained problem of the kind that we propose, for a suitable choice of the initial commitment $\bar{W}_{t_{0}}$. It follows that we can characterize the asymptotic dynamics of tax rates in the model of Chari et al. (which is the main goal of their paper) by characterizing the solution to the constrained policy problem defined here.

An advantage of our reformulation of the optimal policy problem is that the problem that we define - unlike the one that they consider — has as its solution a steady state (or more precisely, a balanced growth path, with constant tax rates) in the case that there are no random fluctuations in either technology or government purchases, if the initial capital stock $k_{t_{0}}$ happens to be consistent with the steady state associated with the initial commitment $\bar{W}_{t_{0}}$. There is furthermore asymptotic convergence to a steady state of this kind under the constrained optimal policy in the case of other (sufficiently nearby) initial conditions. Hence we can characterize the stationary asymptotic fluctuations in tax rates under optimal policy, in the case of small enough shocks, through a local characterization of optimal policy near such a steady state.

Because of this property of optimal dynamics subject to the constraint (1.22), we need not introduce any additional constraints on the admissible range of variation in tax rates (say, an upper bound of 100 percent taxation of income from capital). Assuming initial conditions consistent with a steady state in which such a bound does not bind, the bound will also never bind at any date in the event of small enough shocks, and so we need not consider it at all. If, instead, we were to impose such a bound as an alternative to constraint (1.22) (and the bound were tight enough to preclude the first-best allocation, but loose enough to not bind in the event of zero taxation of capital income), optimal policy would not correspond to a steady state

\footnotetext{
${ }^{10}$ The proof follows the same lines as the proof of Proposition 1 in Benigno and Woodford (2004a), and is omitted here. Chari et al. (1994, p. 625) note that under their formulation of the Ramsey policy problem, the optimal allocation is described by a set of time-invariant allocation rules that apply in each period $t \geq t_{0}+1$, though not in the initial period. Our reformulation of the policy problem makes it clear that the optimal choices $\left(c_{t}, h_{t}, k_{t+1}\right)$ will be time-invariant functions of the state variables $\left(k_{t}, \xi_{t}\right)$.
} 
even in the case of a fully deterministic environment (as the upper bound on the capital tax rate would be initially binding, but never again binding after some finite date), so that the kind of local approximation used below would not be valid even in the case of arbitrarily small shocks and suitably chosen initial conditions.

We now turn to the characterization of optimal steady states for the policy problem just defined.

\subsection{Balanced Growth and Detrending}

Again following Chari et al., we assume that the exogenous disturbances $z_{t}$ and $g_{t}$ each fluctuate around a deterministic trend that grows as $\rho^{t}$, for some $\rho \geq 1$. We assume that preferences are consistent with a balanced growth path; specifically, we assume the functional form

$$
u(c, h)=\frac{\left[c^{1-\gamma}(1-h)^{\gamma}\right]^{\varphi}}{\varphi}
$$

where $0<\gamma<1, \varphi<1, \neq 0$, or (corresponding to the case $\varphi=0$ )

$$
u(c, h)=(1-\gamma) \log c+\gamma \log (1-h)
$$

We also assume a production function of the parametric form

$$
f(k, z h)=k^{\alpha}(z h)^{1-\alpha}
$$

for some $0<\alpha<1$.

We then define detrended versions of the model variables

$$
\tilde{c}_{t} \equiv \frac{c_{t}}{\rho^{t}}, \quad \tilde{y}_{t} \equiv \frac{y_{t}}{\rho^{t}}, \quad \tilde{k}_{t+1} \equiv \frac{k_{t+1}}{\rho^{t}} \quad \tilde{z}_{t} \equiv \frac{z_{t}}{\rho^{t}} \quad \tilde{g}_{t} \equiv \frac{g_{t}}{\rho^{t}} \quad \tilde{b}_{t}^{s} \equiv \frac{b_{t}^{s}}{\rho^{t+1}},
$$

while $h_{t}$ is already a stationary variable without detrending. Given this transformation, it can be shown that the optimal policy problem has the same formulation as presented above except for a redefinition of the discount factor $\beta$, which must be replaced by

$$
\tilde{\beta}=\beta \rho^{\varphi(1-\gamma)},
$$

and a similar redefinition of the depreciation rate $\delta$, which is replaced by

$$
\tilde{\delta} \equiv 1-(1-\delta) \rho^{-1} .
$$


Moreover the production function must be redefined as

$$
\tilde{y}_{t}=\tilde{f}(\tilde{k}, \tilde{z} h) \equiv \rho^{-\alpha} \tilde{k}^{\alpha}(\tilde{z} h)^{1-\alpha} .
$$

In what follows, we drop the tildes (so that " $c_{t}$ " actually refers to $\tilde{c}_{t}$, and so on), but all variables without a tilde should be interpreted as having been appropriately detrended. We preserve the notation $\tilde{\beta}$ and $\tilde{\delta}$ for the alternative numerical coefficients that occur in various equations, as this will be important in calibrating the numerical values of these coefficients.

After this rescaling of variables, (1.18) implies

$$
W_{t} \equiv b_{t-1}^{s} u_{c}\left(c_{t}, h_{t}\right)+u_{c}\left(c_{t}, h_{t}\right) k_{t}\left[(1-\tilde{\delta})+\left(1-\tau_{t}^{k}\right) f_{k}\left(z_{t} h_{t}, k_{t}\right)+\delta \rho^{-1} \tau_{t}^{k}\right]
$$

where we note that

$$
\delta \rho^{-1}=\rho^{-1}-(1-\tilde{\delta})
$$

while (1.20) becomes

$$
u_{c}\left(c_{t}, h_{t}\right)=\tilde{\beta} E_{t}\left\{u_{c}\left(c_{t+1}, h_{t+1}\right)\left[(1-\tilde{\delta})+\left(1-\tau_{t+1}^{k}\right) f_{k}\left(k_{t+1}, z_{t+1} h_{t+1}\right)+\delta \rho^{-1} \tau_{t+1}^{k}\right]\right\} .
$$

\subsection{Optimal Steady States}

Here we show the existence of a steady state, i.e., of an optimal policy (under appropriate initial conditions) of the above policy problem that involves constant values of all stationary variables. We now consider a deterministic problem in which the detrended exogenous disturbances $z_{t}, g_{t}$, each take constant values $\bar{z}>0$ and $\bar{g} \geq 0$ for all $t \geq t_{0}$, and we start from initial conditions $b_{t_{0}-1}^{s}=\bar{b}>0$ and $k_{t_{0}}=\bar{k}>0$. Given any initial detrended debt $\bar{b}$, we wish to find initial commitments $\bar{W}_{t_{0}}=\bar{W}$ and an initial detrended capital stock $\bar{k}>0$ such that the optimal plan involves a constant policy $\overline{\tau^{h}}, \overline{\tau^{k}}, \bar{c}, \bar{h}, \bar{k}, \bar{b}$ and $W_{t}=\bar{W}$, each period, where $\bar{b}, \bar{k}$ and $\bar{W}$ coincide with the initial conditions.

The optimization problem can be equivalently written as choosing the sequences $\left\{c_{t}, h_{t}, k_{t+1}\right\}_{t=t_{0}}^{\infty}$ that maximize

$$
U_{t_{0}}=\sum_{T=t_{0}}^{\infty} \tilde{\beta}^{T-t_{0}} u\left(c_{T}, h_{T}\right)
$$


subject to the sequence of constraints

$$
f\left(k_{t}, z_{t} h_{t}\right)=c_{t}+g_{t}+k_{t+1}-(1-\tilde{\delta}) k_{t}
$$

for each $t \geq t_{0}$, given the initial condition $k_{t_{0}}>0$, and the additional constraint $W_{t_{0}}=\bar{W}$, where

$$
W_{t_{0}}=\sum_{T=t_{0}}^{\infty} \tilde{\beta}^{T-t_{0}}\left[u_{c}\left(c_{T}, h_{T}\right) c_{T}+u_{h}\left(c_{T}, h_{T}\right) h_{T}\right] .
$$

The first-order conditions with respect to $c_{t}$ take the form

$$
u_{c}\left(c_{t}, h_{t}\right)-\left[u_{c}\left(c_{t}, h_{t}\right)+u_{c c}\left(c_{t}, h_{t}\right) c_{t}+u_{h c}\left(c_{t}, h_{t}\right) h_{t}\right] \lambda_{0}-\lambda_{1, t}=0
$$

for each $t \geq t_{0}$, where $\lambda_{0}$ is the Lagrange multiplier associated with the constraint on $W_{t_{0}}$ and $\left\{\lambda_{1, t}\right\}$ is the sequence of multipliers associated with the sequence of constraints (1.30). The first-order condition with respect to $h_{t}$ can similarly be written

$$
\begin{aligned}
0= & u_{h}\left(c_{t}, h_{t}\right)-\left[u_{c h}\left(c_{t}, h_{t}\right) c_{t}+u_{h h}\left(c_{t}, h_{t}\right) h_{t}+u_{h}\left(c_{t}, h_{t}\right)\right] \lambda_{0}+ \\
& +\bar{z} f_{h}\left(k_{t}, \bar{z} h_{t}\right) \lambda_{1, t}
\end{aligned}
$$

for each $t \geq t_{0}$. Finally, the first-order condition with respect to $k_{t+1}$ is given by

$$
\lambda_{1, t}-\tilde{\beta}\left[f_{k}\left(k_{t+1}, \bar{z} h_{t+1}\right)+(1-\tilde{\delta})\right] \lambda_{1, t+1}=0
$$

for each $t \geq t_{0}$.

In a steady-state solution, these conditions respectively reduce to

$$
\begin{gathered}
\bar{u}_{c}(\bar{c}, \bar{h})-\left[\bar{u}_{c}(\bar{c}, \bar{h})+\bar{u}_{c c}(\bar{c}, \bar{h}) \bar{c}+\bar{u}_{h c}(\bar{c}, \bar{h}) \bar{h}\right] \bar{\lambda}_{0}-\bar{\lambda}_{1}=0, \\
\bar{u}_{h}(\bar{c}, \bar{h})-\left[\bar{u}_{c h}(\bar{c}, \bar{h}) \bar{c}+\bar{u}_{h h}(\bar{c}, \bar{h}) \bar{h}+\bar{u}_{h}(\bar{c}, \bar{h})\right] \bar{\lambda}_{0}+\bar{z} f_{h}(\bar{k}, \bar{z} \bar{h}) \bar{\lambda}_{1}=0,
\end{gathered}
$$

and

$$
1=\tilde{\beta}\left[f_{k}(\bar{k}, \bar{z} \bar{h})+(1-\tilde{\delta})\right] .
$$

A first implication of equations (1.37) and (1.7) is that the optimal steady-state tax on capital is zero, as found by Judd (1985) and Chamley (1986).

Equations (1.35), (1.36), (1.37) together with

$$
\bar{c}+\bar{g}+\tilde{\delta} \bar{k}=f(\bar{k}, \bar{z} \bar{h})
$$




$$
\begin{gathered}
(1-\tilde{\beta}) \bar{W}=\bar{u}_{c}(\bar{c}, \bar{h}) \bar{c}+\bar{u}_{h}(\bar{c}, \bar{h}) \bar{h} \\
\bar{W}=\bar{b} \bar{u}_{c}(\bar{c}, \bar{h})+\frac{\bar{u}_{c}(\bar{c}, \bar{h}) \bar{k}}{\tilde{\beta}} .
\end{gathered}
$$

can be solved, given $\bar{b}$, to obtain the steady-state values for $\bar{h}, \bar{c}, \bar{k}, \bar{\lambda}_{0}, \bar{\lambda}_{1}, \bar{W}$. The initial level of debt $\bar{b}$ can be freely chosen, within certain bounds that ensure that the problem has a solution. Finally, the steady-state tax rate on labor is determined by

$$
\frac{\bar{u}_{h}(\bar{c}, \bar{h})}{\bar{u}_{c}(\bar{c}, \bar{h})}=-\left(1-\bar{\tau}^{h}\right) \bar{z} f_{h}(\bar{k}, \bar{z} \bar{h}),
$$

where if $\bar{\tau}^{h}$ is restricted to lie within the range $0 \leq \bar{\tau}^{h} \leq 1$, tighter restrictions on the feasible initial level of debt must be imposed.

\section{Linear-Quadratic Approximations, Correct and Incorrect}

We now consider an approximate characterization of the optimal dynamics of capital and labor income tax rates in the case of small enough shocks, and initial conditions close enough to consistency with an optimal steady state. We first show that a naive approach to LQ approximation would not yield correct results, and then show how a correct LQ approximate problem can be derived.

\subsection{Naive LQ Approximation}

By "naive" LQ approximation we mean an approach that would simply compute a second-order Taylor series expansion of the utility function around the steady-state allocation, and maximize this quadratic objective (or minimize the corresponding quadratic loss function) subject to the linear constraints obtained by log-linearizing the requirements for a rational-expectations equilibrium around that same steadystate allocation. In general, the solution to the LQ problem defined in this way will not be a correct linear approximation to optimal policy, even in the case of small enough shocks and initial conditions close enough to consistency with the steady state around which the Taylor series expansions are computed, for reasons discussed in Judd (1995, 1999), Kim and Henderson (2004, appendix), Kim and Kim (2003), 
Kim et al. (2003), Woodford (2002; 2003, chap. 6), and Benigno and Woodford (2004b).

Suppose that we compute a second-order Taylor series expansion to the utility function (1.1), expanding in powers of log deviations of consumption and hours ${ }^{11}$ from the steady-state levels of these variables, in an optimal steady state of the kind characterized in the previous section. The choice of the particular steady state around which we compute our expansions is arbitrary; the local approximation to optimal policy (if valid at all) will apply to initial conditions close enough to consistency with that particular steady state. In our numerical work, we calibrate the steady state to involve a tax rate on labor income that coincides with the long-run average value in the simulations of Chari et al. (1994).

We show in the appendix that such an approximation takes the form

$U_{t_{0}}=\bar{u}_{c} \bar{c} E_{t_{0}} \sum_{t=t_{0}}^{\infty} \tilde{\beta}^{t-t_{0}}\left\{\hat{c}_{t}-\phi \hat{h}_{t}+\frac{1}{2}\left(1-\sigma^{-1}\right) \hat{c}_{t}^{2}-\frac{1}{2} \phi(1+\nu) \hat{h}_{t}^{2}+\psi \hat{c}_{t} \hat{h}_{t}\right\}+$ t.i.p. $+\mathcal{O}\left(\|\xi\|^{3}\right)$,

where $\hat{c}_{t} \equiv \log \left(c_{t} / \bar{c}\right),{ }^{12} \hat{h}_{t} \equiv \log \left(h_{t} / \bar{h}\right)$; the expression "t.i.p." indicates terms that are independent of policy (because they depend only on the exogenous state of the world), and so irrelevant for welfare comparison of alternative policies; and the residual is of third or higher order in the bound $\|\xi\|$ on the amplitude of the exogenous disturbances, on the assumption that the log deviations $\hat{c}_{t}$ and $\hat{h}_{t}$ are at most of order $\mathcal{O}(\|\xi\|)$, as will be true under optimal policy in the case of initial conditions consistent with the steady state, or deviating from it by an amount that is only of order $\mathcal{O}(\|\xi\|) .{ }^{13}$ In writing the coefficients of this expansion, we have defined $\phi \equiv-\bar{u}_{h} \bar{h} / \bar{u}_{c} \bar{c}, \sigma^{-1} \equiv-\bar{u}_{c c} \bar{c} / \bar{u}_{c}, \nu \equiv \bar{u}_{h h} \bar{h} / \bar{u}_{h}$, and $\psi \equiv \bar{u}_{c h} \bar{h} / \bar{u}_{c} \cdot{ }^{14}$ Thus expected utility $U_{t_{0}}$ varies inversely, in the case of small enough disturbances, with a quadratic

\footnotetext{
${ }^{11}$ Our Taylor series expansion is in terms of the logs of consumption and hours, rather than consumption and hours themselves, because we intend to log-linearize constraints.

${ }^{12}$ It should be recalled that here and below $c_{t}$ actually refers to the detrended level of consumption $\tilde{c}_{t}, k_{t}$ refers to the detrended capital stock $\tilde{k}_{t}$, and so on.

${ }^{13}$ It is important for this last conclusion to be correct that we have chosen an optimal steady state around which to compute our Taylor series expansion.

${ }^{14}$ In the case of the form of preferences (1.24), these coefficients correspond to $\phi=(\gamma / 1-\gamma)(\bar{h} / 1-$ $\bar{h}), \sigma^{-1}=-[(1-\gamma) \varphi-1], \nu=(1-\gamma \varphi)(\bar{h} / 1-\bar{h})$, and $\psi=-\gamma \varphi(\bar{h} / 1-\bar{h})$.
} 
loss function of the form

$$
E_{t_{0}} \sum_{t=t_{0}}^{\infty} \tilde{\beta}^{t-t_{0}} L_{t},
$$

where the period loss function is of the form

$$
L_{t}=\frac{1}{2}\left[\left(\sigma^{-1}-1\right) \hat{c}_{t}^{2}-2 \psi \hat{c}_{t} \hat{h}_{t}+\phi(1+\nu) \hat{h}_{t}^{2}\right]+\phi \hat{h}_{t}-\hat{c}_{t} .
$$

We can log-linearize the constraint (1.30) around the same steady state, obtaining

$$
\hat{k}_{t+1}=\tilde{\beta}^{-1} \hat{k}_{t}-s_{k}^{-1}\left[s_{c} \hat{c}_{t}-(1-\alpha)\left(\hat{z}_{t}+\hat{h}_{t}\right)+s_{g} \hat{g}_{t}\right],
$$

where $\hat{k}_{t} \equiv \log \left(k_{t} / \bar{k}\right), \hat{z}_{t} \equiv \log z_{t}$, and $\hat{g}_{t} \equiv \log \left(g_{t} / \bar{g}\right)$, and in writing the coefficients we define $s_{k} \equiv \bar{k} / \bar{y}, s_{c} \equiv \bar{c} / \bar{y}$, and $s_{g} \equiv \bar{g} / \bar{y}$. A similar log-linearization of (1.31) yields

$$
\tilde{W}_{t_{0}}=E_{t_{0}} \sum_{T=t_{0}}^{\infty} \tilde{\beta}^{T-t_{0}}\left\{d_{c} \hat{c}_{T}+d_{h} \hat{h}_{T}\right\},
$$

where $\tilde{W}_{t_{0}} \equiv\left(\bar{W}_{t_{0}}-\bar{W}\right) / \bar{u}_{c} \bar{c}$ is given as an initial condition, and we define the coefficients $d_{c} \equiv 1-\sigma^{-1}+\psi, d_{h} \equiv \psi-\phi(1+\nu)$. The naive LQ approximate problem would then be to choose sequences $\left\{\hat{c}_{t}, \hat{h}_{t}, \hat{k}_{t+1}\right\}_{t=t_{0}}^{\infty}$ to minimize the loss function defined by $(2.2)-(2.3)$ subject to constraints (2.4) and (2.5), given the initial conditions $\hat{k}_{t_{0}}$ and $\tilde{W}_{t_{0}}$.

The solution to this LQ problem is not generally a correct linear approximation to the optimal policy defined in the previous section. It is especially easy to see this in the case of preferences of the form (1.25), which corresponds to the baseline calibration in Chari et al. (1994). In this case $\sigma=1, \psi=0$, and the naive loss function (2.3) reduces to

$$
L_{t}=\frac{1}{2} \phi(1+\nu) \hat{h}_{t}^{2}+\phi \hat{h}_{t}-\hat{c}_{t}
$$

This loss function would not penalize fluctuations in any endogenous variables other than hours worked.

One can easily show that this LQ problem has a solution in which neither random fluctuations in technology nor in government purchases should be allowed to have any effect on equilibrium hours worked. (If we assume that $\bar{W}_{t_{0}}=\bar{W}$ and that there are no deviations of either $g_{t}$ or $z_{t}$ from their trend values that are forecastable as of date $t_{0}$, then the solution involves $\hat{h}_{t}=0$ for all dates $t \geq t_{0}$.) This is clearly 
possible to achieve through a suitable tax policy, since absolutely any linear responses of the endogenous variables $\left\{\hat{c}_{t}, \hat{h}_{t}\right\}$ to unexpected shocks at dates after $t_{0}$ will be consistent with (2.5), and there will exist paths for $\left\{\hat{c}_{t}, \hat{k}_{t+1}\right\}$ consistent with (2.4) under the assumption that $\hat{h}_{t}=0$ at all dates. Indeed, there will exist an infinity of bounded processes of this kind, for any bounded fluctuations in the disturbances $\left\{\hat{z}_{t}, \hat{g}_{t}\right\}$, including ones in which consumption and capital accumulation respond to arbitrary random events ("sunspots"); and according to the objective defined by (2.2) and (2.6), these processes are all equally optimal.

But it is not hard to show that these are not correct conclusions about the solution to the optimization problem stated in the previous section. For example, consider allocations in which $h_{t}=\bar{h}$ at all dates, and ask whether it is really true that the introduction of random variations in the paths of capital and consumption, of a kind consistent with the feasibility constraint (1.30), in response to sunspot events not forecastable at date $t_{0}$, will have no effect on expected utility, considering only effects of second order in the amplitude of the disturbances. The conclusion is obviously wrong: any such response to sunspot events (even if both the capital stock and consumption remain within intervals of a width that is $\mathcal{O}(\|\xi\|)$ ) will lower expected utility, and by an amount that is of second order, owing both to the concavity of utility in consumption and to the concavity of the production function in capital. One can similarly show, by substituting a constant number of hours into the first-order conditions for Ramsey policy presented in section 1.4 above, that these conditions cannot be jointly satisfied by any policy that keeps hours constant; and furthermore one or more equations must have a discrepancy that is of order $\mathcal{O}(\|\xi\|)$, so that this is not even a correct first-order approximation to the optimal labor allocation.

The problem with the naive LQ analysis is that while (2.1) does give a correct approximation to expected utility, that is accurate to second order if all terms are evaluated under a candidate policy in a way that is accurate to second order, a solution for the paths $\left\{\hat{c}_{t}, \hat{h}_{t}\right\}$ under a given policy that is accurate only to first order will not suffice to allow a sufficiently accurate evaluation of the linear terms in (2.1). And while the log-linearized constraint (2.4) correctly indicates to first order the available tradeoffs between fluctuations in consumption, hours, and capital, it omits secondorder terms. For example, in the case that $\left\{k_{t+1}\right\}$ varies in response to unforecastable sunspot events while $h_{t}$ remains constant, as in the thought experiment of the previous paragraph, the path $\left\{\hat{c}_{t}\right\}$ implied by (2.4) omits negative second-order effects on the 
expected level of log consumption that occur as a result of the concavity of both utility (i.e., of the log function) and of the production function. But these omitted secondorder effects may affect expected utility as much as do the included $E_{t_{0}} \hat{h}_{t}^{2}$ terms, even if the amplitude of the fluctuations is made arbitrarily small. Thus the LQ analysis gives an incorrect welfare ranking of alternative possible patterns of response of the endogenous variables to random disturbances, even when the disturbances are small.

\subsection{An Alternative Quadratic Objective}

One way of correcting the problem just illustrated would be to approximate the constraints to an accuracy that is at least second order in the amplitude of departures from the steady-state allocation. This can be done, for example, using a higher-order Taylor series expansion of the exact conditions; but this would mean abandoning the convenience of an LQ framework for the approximate policy problem. Alternatively, we can replace the quadratic objective derived in the previous section by an alternative function, that also approximates expected utility to second order, ${ }^{15}$ and that involves no linear terms of the kind present in (2.1). It is then possible to evaluate the quadratic objective to second order using only a characterization of equilibrium fluctuations that is accurate to first order. In Benigno and Woodford (2004b), we show that it is quite generally possible to derive a quadratic approximation to utility with this property, as long as the steady state around which one expands is itself optimal - i.e., it represents optimal policy in the case that there are no random disturbances, as is the case in our example. Here we illustrate how this is possible in the present problem.

We begin with the second-order Taylor series expansion for utility (2.1), which can be written in a more compact way as

$$
U_{t_{0}}=\bar{u}_{c} \bar{c} E_{t_{0}} \sum_{t=t_{0}}^{\infty} \tilde{\beta}^{t-t_{0}}\left\{a_{x}^{\prime} x_{t}-\frac{1}{2} x_{t}^{\prime} A_{x} x_{t}\right\}+\text { t.i.p. }+\mathcal{O}\left(\|\xi\|^{3}\right)
$$

\footnotetext{
${ }^{15}$ Of course, since the functions are different, they are not equal to second order in the case of all possible paths for the endogenous variables. However, they may be equal in the case of all paths that represent possible equilibria. The representation (2.1) is the unique approximation that would be accurate to second order in the case of arbitrary small departures from the steady state, even those that are not possible under any tax policy — but that property is stronger than is necessary for our purposes.
} 
where the vector $x_{t}$ is defined as $x_{t}^{\prime} \equiv\left[\begin{array}{ll}c_{t} & h_{t}\end{array}\right]$ and

$$
\begin{gathered}
a_{x} \equiv\left[\begin{array}{c}
1 \\
-\phi
\end{array}\right], \\
A_{x} \equiv\left[\begin{array}{cc}
-\left(1-\sigma^{-1}\right) & -\psi \\
-\psi & \phi(1+\nu)
\end{array}\right] .
\end{gathered}
$$

We now take a second-order approximation of equation (1.30). As shown in the appendix we obtain

$$
\begin{aligned}
0= & \sum_{t=t_{0}}^{\infty} \tilde{\beta}^{t-t_{0}}\left\{s_{c} \hat{c}_{t}-(1-\alpha) \hat{h}_{t}+\frac{1}{2} s_{c} \hat{c}_{t}^{2}+\frac{1}{2} \alpha(1-\alpha) \hat{\tilde{k}}_{t}^{2}+\right. \\
& \left.-\frac{1}{2}(1-\alpha)\left(\hat{h}_{t}^{2}+2 \hat{z}_{t} \hat{h}_{t}\right)\right\}+ \text { t.i.p. }+\mathcal{O}\left(\|\xi\|^{3}\right),
\end{aligned}
$$

where we have defined $\hat{\tilde{k}}_{t} \equiv \hat{k}_{t}-\hat{h}_{t}-\hat{z}_{t}$. We can write (2.8) as

$$
0=\sum_{t=t_{0}}^{\infty} \tilde{\beta}^{t-t_{0}}\left\{b_{x}^{\prime} x_{t}+\frac{1}{2} x_{t}^{\prime} B_{x} x_{t}+x_{t}^{\prime} B_{\xi} \xi_{t}+\frac{1}{2} \alpha(1-\alpha) \hat{\tilde{k}}_{t}^{2}\right\}+\text { t.i.p. }+\mathcal{O}\left(\|\xi\|^{3}\right),
$$

where now

$$
\begin{gathered}
b_{x} \equiv\left[\begin{array}{c}
s_{c} \\
-(1-\alpha)
\end{array}\right], \\
B_{x} \equiv\left[\begin{array}{cc}
s_{c} & 0 \\
0 & -(1-\alpha)
\end{array}\right], \\
B_{\xi} \equiv\left[\begin{array}{cc}
0 & 0 \\
-(1-\alpha) & 0
\end{array}\right],
\end{gathered}
$$

where we have used the definition $\xi_{t}^{\prime}=\left[\begin{array}{ll}\hat{z}_{t} & \hat{g}_{t}\end{array}\right]$.

The final expression that we need to approximate is (1.31). As it is shown in the appendix a second-order approximation yields to

$$
\begin{aligned}
\tilde{W}_{t_{0}}= & E_{t_{0}} \sum_{t=t_{0}}^{\infty} \tilde{\beta}^{t-t_{0}}\left\{\left(1-\sigma^{-1}+\psi\right) \hat{c}_{t}+(\psi-\phi-\phi \nu) \hat{h}_{t}\right. \\
& +\frac{1}{2}\left(\psi+\psi \psi_{1}-\phi-\phi \nu-\phi \nu \nu_{1}-2 \phi \nu\right) \hat{h}_{t}^{2}+ \\
& +\frac{1}{2}\left(1-\sigma^{-1}+\psi-\sigma^{-1} \sigma_{1}^{-1}-\sigma^{-1} \psi_{2}-2 \sigma^{-1}\right) \hat{c}_{t}^{2}+ \\
& \left.+\left(\psi \psi_{1}-\sigma^{-1} \psi_{2}+2 \psi\right) \hat{c}_{t} \hat{h}_{t}\right\} \\
& + \text { t.i.p. }+\mathcal{O}\left(\|\xi\|^{3}\right) .
\end{aligned}
$$


where we have defined $\sigma_{1}^{-1} \equiv \bar{u}_{c c c} \bar{c} / \bar{u}_{c c}, \psi_{1} \equiv \bar{u}_{c h h} \bar{h} / \bar{u}_{c h}, \psi_{2} \equiv \bar{u}_{c c h} \bar{h} / \bar{u}_{c c}, \nu_{1} \equiv$ $\bar{u}_{h h h} \bar{h} / \bar{u}_{h h}$ and $\tilde{W}_{t} \equiv\left(W_{t_{0}}-\bar{W}\right) / \bar{u}_{c} \bar{c}$. Finally we can write $(2.10)$ as

$$
\tilde{W}_{t_{0}}=E_{t_{0}} \sum_{t=t_{0}}^{\infty} \tilde{\beta}^{t-t_{0}}\left\{c_{x}^{\prime} x_{t}+\frac{1}{2} x_{t}^{\prime} C_{x} x_{t}+x_{t}^{\prime} C_{\xi} \xi_{t}\right\}+\text { t.i.p. }+\mathcal{O}\left(\|\xi\|^{3}\right)
$$

where now

$$
\begin{gathered}
c_{x} \equiv\left[\begin{array}{cc}
\left(1-\sigma^{-1}+\psi\right) \\
(\psi-\phi-\phi \nu)
\end{array}\right] \\
C_{x} \equiv\left[\begin{array}{rr}
\left(1-\sigma^{-1}+\psi-\sigma^{-1} \sigma_{1}^{-1}-\sigma^{-1} \psi_{2}-2 \sigma^{-1}\right) & \left(\psi \psi_{1}-\sigma^{-1} \psi_{2}+2 \psi\right) \\
\left(\psi \psi_{1}-\sigma^{-1} \psi_{2}+2 \psi\right) & \left(\psi+\psi \psi_{1}-\phi-\phi \nu-\phi \nu \nu_{1}-2 \phi \nu\right)
\end{array}\right], \\
C_{\xi} \equiv\left[\begin{array}{cc}
0 & 0 \\
0 & 0
\end{array}\right]
\end{gathered}
$$

We can now use an appropriate linear combination of (2.9) and (2.11) to obtain an expression with linear terms that exactly cancel those in (2.7). Using this expression to subsitute for the linear terms in (2.7), we obtain

$$
U_{t_{0}}=-\bar{u}_{c} \bar{c} E_{t_{0}} \sum_{t=t_{0}}^{\infty} \tilde{\beta}^{t-t_{0}}\left\{\frac{1}{2} x_{t}^{\prime} Q_{x} x_{t}+x_{t}^{\prime} Q_{\xi} \xi_{t}+\frac{1}{2} q_{k} \hat{\tilde{k}}_{t}^{2}\right\}+\bar{u}_{c} \bar{c} \vartheta_{2} \tilde{W}_{t_{0}}+\text { t.i.p. }+\mathcal{O}\left(\|\xi\|^{3}\right)
$$

where

$$
\begin{gathered}
Q_{x} \equiv A_{x}+\vartheta_{1} B_{x}+\vartheta_{2} C_{x} \\
Q_{\xi} \equiv \vartheta_{1} B_{\xi}+\vartheta_{2} C_{\xi} \\
q_{k} \equiv \alpha(1-\alpha) \vartheta_{1}
\end{gathered}
$$

and

$$
\begin{aligned}
& \vartheta_{1}=\frac{-\phi\left(\sigma^{-1}+\nu\right)+\psi(\phi+1)}{\psi\left[(1-\alpha)+s_{c}\right]+\bar{\tau}^{h}(1-\alpha)(1+\nu)-(1-\alpha)\left(\sigma^{-1}+\nu\right)}, \\
& \vartheta_{2}=\frac{\bar{\tau}^{h}(1-\alpha)}{\psi\left[(1-\alpha)+s_{c}\right]+\bar{\tau}^{h}(1-\alpha)(1+\nu)-(1-\alpha)\left(\sigma^{-1}+\nu\right)} .
\end{aligned}
$$

We note that for low values of $\psi$ and $\bar{\tau}^{h}$, it is likely that $\vartheta_{1}>0$ and $\vartheta_{2}<0$. We also note that the term $\vartheta_{2} \tilde{W}_{t_{0}}$ is independent of policy, because of the initial commitment regarding the value of $\tilde{W}_{t_{0}}$. 
It then follows that maximization of expected utility is equivalent, to second order, to the minimization of an objective of the form (2.2), where the period loss function is now of the form

$$
L_{t}=\frac{1}{2} x_{t}^{\prime} Q_{x} x_{t}+x_{t}^{\prime} Q_{\xi} \xi_{t}+\frac{1}{2} q_{k} \hat{\tilde{k}}_{t}^{2}
$$

where we define

$$
\hat{\tilde{k}}_{t}=\hat{k}_{t}-\left(\hat{z}_{t}+\hat{h}_{t}\right)
$$

and the coefficients are given by

$$
\begin{gathered}
Q_{x}=\left[\begin{array}{ll}
q_{x, 11} & q_{x, 12} \\
q_{x, 21} & q_{x, 22}
\end{array}\right], \\
Q_{\xi}=\left[\begin{array}{cc}
0 & 0 \\
q_{\xi, 21} & 0
\end{array}\right] .
\end{gathered}
$$

This can be written more extensively as

$$
L_{t}=\frac{1}{2}\left\{q_{x, 11} \hat{c}_{t}^{2}+2 q_{x, 12} \hat{c}_{t} \hat{h}_{t}+q_{x, 22} \hat{h}_{t}^{2}+2 \hat{h}_{t} q_{\xi, 21} \hat{z}_{t}+q_{k} \hat{\tilde{k}}_{t}^{2}\right\}
$$

or alternatively as

$$
L_{t}=\frac{1}{2}\left\{q_{c}\left(\hat{c}_{t}-\theta \hat{h}_{t}\right)^{2}+q_{h}\left(\hat{h}_{t}-h_{t}^{*}\right)^{2}+q_{k} \hat{\tilde{k}}_{t}^{2}\right\}
$$

where we have defined $q_{c} \equiv q_{x, 11}, \theta \equiv-q_{x, 12} / q_{x, 11}, q_{h} \equiv q_{x, 22}-q_{x, 12}^{2} / q_{x, 11}$, and $h_{t}^{*} \equiv \theta_{z} \hat{z}_{t}$, in which expression $\theta_{z} \equiv-q_{h}^{-1} q_{\xi, 21}$.

Expression (2.13) is now a quadratic loss function with no linear terms, as desired. In the appendix, we present the general conditions under which this function is convex, at least on the linear subspace of sequences consistent with our log-linear constraints. (Convexity in this sense ensures that the first-order conditions for the LQ problem defined in the next section characterize a loss minimum, and hence a welfare maximum.) Here it suffices to note that sufficient conditions for convexity are that the coefficients $q_{c}, q_{h}, q_{k}$ all be positive; in the numerical examples considered in the next section, this is always the case.

It follows that the objective of policy can be understood as a combination of three distinct stabilization goals. The first is stabilization of a linear combination of consumption and hours; the second is stabilization of hours around a level that depends on the current state of productivity; and the third is stabilization of the 
capital stock per unit of effective labor supply (i.e., labor supply in efficiency units). Optimal policy will generally involve some tradeoff among these three goals. Note that the quadratic loss function obtained in this way is quite different from the function (2.3) obtained from a simple Taylor series expansion of the utility function.

\subsection{A Correct LQ Approximation}

Because (2.13) contains no linear terms, this loss function can be evaluated to second order using only a log-linear approximation to the equilibrium evolution of the endogenous variables under a given policy. It follows that we may represent the constraints upon the outcomes achievable under alternative policies, to a sufficient degree of accuracy, using only log-linear approximations to the structural equations that must be satisfied in a rational-expectations equilibrium.

Our approximate LQ problem is then to choose sequences $\left\{\hat{c}_{t}, \hat{h}_{t}, \hat{k}_{t+1}\right\}_{t=t_{0}}^{\infty}$ to minimize the loss function defined by (2.2) and (2.13) subject to the constraints (2.4) and (2.5), given the initial conditions $\hat{k}_{t_{0}}$ and $\tilde{W}_{t_{0}}$. The solution to this problem will be given by a set of time-invariant policy rules if $\tilde{W}_{t_{0}}$ is chosen in a suitable way as a function of the values of $\hat{k}_{t_{0}}, \hat{z}_{t_{0}}$, and $\hat{g}_{t_{0}}$ - specifically, if the initial commitment is the same function of the initial state as the corresponding commitments made at later dates will be of the economy's state at those later dates. One simple way to ensure this is to specify $\tilde{W}_{t_{0}}$ to take the value such that the constraint (2.5) does not bind - i.e., such that the solution processes $\left\{\hat{c}_{t}, \hat{h}_{t}, \hat{k}_{t+1}\right\}$ are the same as if this constraint were omitted. This is the specification of $\tilde{W}_{t_{0}}$ that we shall choose. The approximate LQ problem can then be stated more simply as the choice of sequences $\left\{\hat{c}_{t}, \hat{h}_{t}, \hat{k}_{t+1}\right\}_{t=t_{0}}^{\infty}$ to minimize the loss function defined by (2.2) and (2.13) subject to the constraint (2.4), given the initial condition $\hat{k}_{t_{0}}$.

Once we have solved for the optimal allocation $\left\{\hat{c}_{t}, \hat{h}_{t}, \hat{k}_{t+1}\right\}$, the implied path for the state-contingent commitments $\tilde{W}_{t} \equiv\left(\bar{W}_{t}-\bar{W}\right) / \bar{u}_{c} \bar{c}$ each period is given by

$$
\tilde{W}_{t}=E_{t} \sum_{T=t}^{\infty} \tilde{\beta}^{T-t_{0}}\left\{d_{c} \hat{c}_{T}+d_{h} \hat{h}_{T}\right\},
$$

which represents a log-linear approximation to (1.19). (The coefficients are the same as in (2.5), which is a special case of this equation.) The implied value of the public debt $\hat{b}_{t}^{s} \equiv \log \left(b_{t}^{s} / \bar{b}\right)$ each period can then be obtained by solving

$$
\tilde{\beta}^{-1} s_{k}\left[\hat{k}_{t+1}+E_{t}\left(-\sigma^{-1} \hat{c}_{t+1}+\psi \hat{h}_{t+1}\right)\right]=s_{c} E_{t} \tilde{W}_{t+1}-s_{b}\left[\hat{b}_{t}^{s}+E_{t}\left(-\sigma^{-1} \hat{c}_{t+1}+\psi \hat{h}_{t+1}\right)\right],
$$


which represents a log-linear approximation to (1.21), where $s_{b} \equiv \bar{b} / \bar{y}$. The implied capital income tax rate $\hat{\tau}_{t}^{k} \equiv-\log \left(1-\tau_{t}^{k}\right)$ each period is similarly obtained by solving

$$
s_{c} \tilde{W}_{t}=s_{b} \hat{b}_{t-1}^{s}-b_{c} \hat{c}_{t}+b_{h} \hat{h}_{t}+b_{k} \hat{k}_{t}+s_{c}^{-1} \alpha(1-\alpha) \hat{z}_{t}-b_{\tau} \tau_{t}^{k}
$$

a log-linear approximation to (1.18), where we define the coefficients $b_{c} \equiv s_{b} \sigma^{-1}+$ $\tilde{\beta}^{-1} s_{k} \sigma^{-1}, b_{h} \equiv s_{b} \psi+\tilde{\beta}^{-1} s_{k} \psi+\alpha(1-\alpha), b_{k} \equiv \tilde{\beta}^{-1} s_{k}-\alpha(1-\alpha)$, and $b_{\tau} \equiv s_{k}\left(\tilde{\beta}^{-1}-\rho^{-1}\right)$.

Finally, we can obtain the optimal path of the tax on labor income by using a log-linear approximation of (1.8) in which (1.11) is used to substitute for $w_{t}$. This yields

$$
\hat{\tau}_{t}^{h}=\hat{z}_{t}+\alpha \hat{\tilde{k}}_{t}-\left(\sigma^{-1}-\phi^{-1} \psi\right) \hat{c}_{t}-(\nu-\psi) \hat{h}_{t}
$$

In this way, we obtain log-linear equations for the optimal responses of both tax rates to exogenous disturbances.

\section{Optimal Policy: Numerical Results}

We now illustrate the results that we obtain from a numerical application of the above method, when the model is parameterized as in Chari et al. (1994). Comparing our results to those that they obtain using an alternative numerical method will provide one way of judging the numerical accuracy of the LQ approximation.

\subsection{Parameter Values}

Following Chari et al. (1994), we assign the parameters $\alpha, \tilde{\beta}, \gamma, \tilde{\delta}, \rho$, and $\bar{g}$ the values listed in Table 1. ${ }^{16}$ Chari et al. consider the effects of alternative values for the preference parameter $\varphi$ (their $\psi$ ); like them, we give primary emphasis to the two cases $\varphi=0$ (the "baseline" case) and $\varphi=-8$ (the "high risk-aversion" case). As shown in the second panel of Table 1 , we obtain different steady-state values for a number of variables in the two cases.

\footnotetext{
${ }^{16}$ The exact values used for $\alpha, \tilde{\delta}$ and $\bar{g}$ are actually longer decimals, supplied by Larry Christiano, but rounded to three digits in the table. The same is true of the values given below for $\sigma_{g}$ and $\sigma_{z}$. The value here used for $\bar{g}$ assumes a supply of hours (to be used either for work or leisure) that is normalized to equal 1 , and a steady-state value for the detrended productivity factor $\bar{z}$ that is normalized to equal 1.
} 
We have shown above that an optimal steady state necessarily involves $\bar{\tau}^{k}=0$; however, given the model parameters, there is a continuum of steady states with different steady-state tax rates on labor income, corresponding to different choices for the value of initial private wealth $\bar{W}_{t_{0}}$ (and correspondingly different steady-state levels of public debt). We specify $\bar{W}$ in the case of each choice of the preference parameter $\varphi$ so that the resulting optimal steady state corresponds to the steady state around which the dynamics fluctuate asymptotically in the "baseline" and "high riskaversion" simulations of Chari et al. (1994) respectively. ${ }^{17}$ In our calculations, we do this by specifying the steady-state labor tax rate $\bar{\tau}^{h}$ to equal the long-run average labor tax rate reported by Chari et al. (1994, Table 2) for those two simulations, and then inferring the steady-state values $\bar{k}, \bar{y}, \bar{W}, \bar{b}^{s}$, and so on implied by these choices. The steady-state values of several variables that are important for the derivation of our LQ approximate policy problem are shown in the second panel of Table 2, in the two columns corresponding to the two alternative specifications of $\varphi$ and $\bar{\tau}^{h}$.

These values are obtained as follows. The steady-state relations (1.37) and (1.38) imply respectively that the shares $s_{c}, s_{g}, s_{k}$ must satisfy the two relations

$$
\begin{gathered}
(1-\tilde{\delta})+\alpha s_{k}^{-1}=\tilde{\beta}^{-1}, \\
s_{c}+s_{g}+\tilde{\delta} s_{k}=1 .
\end{gathered}
$$

Steady-state relation (1.41) implies that

$$
\phi=\left(1-\bar{\tau}^{h}\right)(1-\alpha) s_{c}^{-1},
$$

while our assumed form for the utility function implies that

$$
\phi=\frac{\gamma}{1-\gamma} \frac{\bar{h}}{1-\bar{h}} .
$$

Finally, the production function (1.26) implies that

$$
\frac{\bar{y}}{\bar{h}}=\left(\rho^{-1} s_{k}\right)^{\frac{\alpha}{1-\alpha}}
$$

\footnotetext{
${ }^{17}$ Because Chari et al. do not impose an initial constraint of a kind that makes their optimal policy problem recursive, and do not start from initial conditions consistent with the long-run steady state, their simulations eventually fluctuate around a steady state with a level of public debt different from the level that they assume as an initial condition, and the extent to which this is true depends on the preference parameter $\varphi$. Because we wish to characterize the asymptotic fluctuations and to compare our results to those that they report, we assume a different steady-state public debt in the two cases.
} 
using the normalization $\bar{z}=1$. Combining equations (3.2) - (3.5), we can solve for the steady-state output level

$$
\bar{y}=\frac{\frac{\gamma}{1-\gamma} \frac{1}{\left(1-\bar{\tau}^{h}\right)(1-\alpha)} \bar{g}+\left(\rho^{-1} s_{k}\right)^{\frac{\alpha}{1-\alpha}}}{\left(1+\frac{\gamma}{1-\gamma} \frac{\left(1-\tilde{\delta} s_{k}\right)}{\left(1-\bar{\tau}^{h}\right)(1-\alpha)}\right)} .
$$

Given the calibrated parameter values from the first panel of Table 1, (3.1) allows us to determine the implied value for $s_{k}$. This value, together with the assumed value of $\bar{\tau}^{h}$ (which varies for the two cases considered in the second panel of the table), allows us to solve for steady-state output $\bar{y}$, and hence for $s_{g}$. We can then solve (3.2) for the implied value of $s_{c}$, (3.3) for the implied value of $\phi$, and then (3.4) for the implied value of $\bar{h}$. Once we have determined the steady-state values of these variables, we can similarly solve for the implied values of variables such as $\bar{W}$ and $\bar{b}^{s}$, though these do not matter for the calculations reported below.

We specify each of the two exogenous disturbance processes $x=\hat{z}, \hat{g}$ as a stationary $\mathrm{AR}(1)$ process

$$
x_{t}=\rho_{x} x_{t-1}+\epsilon_{t}^{x}
$$

where $0 \leq \rho_{x}<1$ and $\left\{\epsilon_{t}^{x}\right\}$ is an i.i.d. random variable with bounded support; the two innovation processes are furthermore assumed to be independent of one another. Like Chari et al. (1994, 1995), we parameterize the disturbance processes so as to match both the standard deviation $\sigma_{x}$ and the coefficient of autocorrelation $\rho_{x}$ of the empirical measures of these disturbances discussed in Christiano and Eichenbaum (1992); the values of these moments that we match are given in the third panel of Table 1. Unlike Chari et al., we do not use a numerical method that requires us to discretize our disturbance processes (they assume two-state Markov chains for each disturbance), and so we adopt a convenient $\operatorname{AR}(1)$ specification. ${ }^{18}$ To be specific, in our numerical simulations we assume that $\epsilon_{t}^{x}$ takes each period either the value $+\delta_{x}$ or the value $-\delta_{x}$, with equal probability, where $\delta_{x} \equiv\left(\left(1-\rho_{x}^{2}\right) \sigma_{x}^{2}\right)^{1 / 2}$.

\footnotetext{
${ }^{18}$ This has no consequences at all for the computation of first and second moments implied by our LQ approximation, or alternatively by log-linearization of the conditions that characterize Ramsey policy. The exact law of motion of the disturbances may affect our second-order perturbation calculations, but we find in any event that the second-order corrections are small. The assumed disturbance processes (3.7) allow our model to fall within the class of models treated by SchmittGrohé and Uribe (2004a), so that we can employ their computer code to compute the second-order approximate solution discussed below.
} 
It might appear from the above that our method cannot be applied without first performing the calculations undertaken by Chari et al., which are used as the source of the steady-state values for $\bar{\tau}^{h}$. But we choose this parameter in this way because we wish to examine the extent to which our method results in a similar characterization of the optimal stationary (asymptotic) fluctuations in tax rates as does theirs, in the case that the initial commitment in our policy problem is defined so as to imply the same long-run steady state (in the absence of shocks) as in the policy problem that they consider. (This last qualification is necessary because the present model - unlike the one considered in Benigno and Woodford, 2004a, for example - allows a continuum of optimal steady states, to different ones of which the optimal dynamics converge in the case of different initial conditions.) If we were simply interested in characterizing optimal tax policy for an economy such as the U.S., starting from historically given initial conditions, this way of determining $\bar{\tau}^{h}$ would not be necessary. We could, for example, assign $\bar{W}$ the value that would correspond to the value that private wealth would be expected to have if current U.S. policy were to continue, and determine the optimal steady state consistent with this. This would correspond to somewhat higher values for $\bar{\tau}^{h}$ than those reported in Table 1 and used in the calculations below.

\subsection{Optimal Fluctuations in Tax Rates}

The parameter values reported in Table 1 allow us to compute both the coefficients of the quadratic loss function (2.13) and the coefficients of the log-linearized constraints. The coefficients of the loss function for each of the two cases considered in the middle panel of Table 1 are given in Table 2 . We note that in each case, $q_{c}, q_{h}, q_{k}>0$, so that the loss function is obviously convex and the second-order conditions for loss minimization are satisfied.

It is then straightforward to characterize the optimal linear decision rules for this problem (though we omit the algebra here). We obtain a linear equation of the form $\hat{k}_{t+1}=\Gamma_{k} v_{t}$, where $v_{t}$ is the state vector $\left[\hat{k}_{t} \hat{z}_{t} \hat{g}_{t}\right]^{\prime}$. This equation together with the specification (3.7) for the disturbance processes then completely defines the law of motion of the state vector $\left\{v_{t}\right\}$. We also obtain linear equations $\hat{c}_{t}=\Gamma_{c} v_{t}, \hat{h}_{t}=\Gamma_{h} v_{t}$ for the rest of the optimal resource allocation, so that the dynamics of consumption and hours are also completely described. Substitution of these solutions into equations 
(2.14) - (2.17) then allows us to obtain linear solutions of the form

$$
\hat{\tau}_{t}^{h}=\Gamma_{\tau}^{h} v_{t}, \quad \hat{\tau}_{t}^{k}=\Gamma_{\tau}^{k}(0) v_{t}+\Gamma_{\tau}^{k}(1) v_{t-1}
$$

for the optimal dynamics of the tax rates.

From the log-linear dynamics implied by these equations, we can compute the implied first and second moments of the tax-rate processes reported by Chari et al. (1994, Table 2), which we present in Table 3. As in the table of Chari et al., the columns of our table refer to five separate cases. The first column is the baseline case, in which $\varphi=0$ and the two disturbance processes are parameterized in the way described in Table 1. The second column is the high risk-aversion case, in which instead $\varphi=-8$. In the third column, we again assume $\varphi=0$, but now that there are no variations in government purchases (i.e., we set $\sigma_{g}=0$ ); in the fourth column, assumptions are again as in the baseline case, except that there are no variations in the rate of technical progress (i.e., we set $\sigma_{z}=0$ ). Finally, in the fifth column, $\varphi=0$, and both kinds of random disturbances exist, but we assume that neither disturbance is serially correlated (i.e., we assume the values given in Table 1 for $\sigma_{z}$ and $\sigma_{g}$, but we set $\left.\rho_{z}=\rho_{g}=0\right){ }^{19}$

Like Chari et al., we report statistics regarding the optimal fluctuations in the labor income tax rate $\tau_{t}^{h}$, the ex post capital income rate $\tau_{t}^{k}$, and an "ex ante tax rate for capital income" defined $\mathrm{as}^{20}$

$$
\theta_{t}^{e} \equiv \frac{E_{t}\left[u_{c}\left(c_{t+1}, h_{t+1}\right) \tau_{t+1}^{k}\left(f_{k}\left(k_{t+1}, z_{t+1} h_{t+1}\right)-\delta \rho^{-1}\right)\right]}{E_{t}\left[u_{c}\left(c_{t+1}, h_{t+1}\right)\left(f_{k}\left(k_{t+1}, z_{t+1} h_{t+1}\right)-\delta \rho^{-1}\right)\right]}
$$

The means and standard deviations of all tax rates are reported in percentage points (i.e., we actually report $100 E\left(\tau_{t}^{h}\right)$, etc.). Given the linearity of the policy rules that solve the LQ problem, we are able to obtain analytical results for these moments as functions of model parameters (including the parameters of the disturbance processes), rather than computing sample statistics from a stochastic simulation, as in Chari et al.

\footnotetext{
${ }^{19}$ In simulations 3 through 5 , we choose the same value for $\bar{\tau}^{h}$ as in the baseline case, though the average labor tax rates reported by Chari et al. in these cases are slightly different (see Table 4 below). Because the long-run steady state should have been the same in these cases as in the first column, in the absence of disturbances, we have computed our local expansions around the same steady state in these cases.

${ }^{20}$ This definition follows Chari et al. (1994, p. 627). In our expression of the formula here, $c_{t}$ refers to the detrended consumption level $\tilde{c}_{t}$, and so on.
} 
Most of our statistics are quite similar to those reported by Chari et al. (1994), which we reproduce in Table $4 .^{21}$ The most visible difference is in the average level of the tax rate on capital income; while our method and theirs give quite similar results regarding the mean "ex ante tax rate" defined in (3.9), the ex post tax rate varies so much that even modest non-linearities in its response to shocks do have a non-negligible effect on the mean ex post rate. ${ }^{22}$ Our conclusions about the degree of optimal variation in capital and labor income tax rates, and the degree to which they should co-vary with each of the disturbances are essentially the same as those obtained by Chari et al., and so we do not discuss them further.

We note, however, that there are a number of advantages of our approach to calculations of this kind. One is that our LQ formulation makes it easy to check the second-order conditions for optimality of the policy that satisfies our first-order conditions; this is not addressed by Chari et al., who simply compute an approximate solution to the first-order conditions that characterize Ramsey policy. Another is that our approach is easily extended to deal with more complex specifications, with little increase in computational complexity. For example, allowing for higher-order autocorrelation of the disturbances would be easy in the context of our LQ methodology, and our specification of the disturbances as linear autoregressive processes also makes it straightforward to parameterize these processes on the basis of empirical estimates.

\subsection{Accuracy of the LQ Approximation}

As we have explained (on the basis of more detailed discussion in Benigno and Woodford, 2004b), the solution to the LQ approximate problem derived here yields a correct local linear approximation to the exact decision rules that characterize optimal policy, that should allow a computation of statistics like those presented in Table 3 that is of arbitrary accuracy in the case that the disturbances are small enough in amplitude.

\footnotetext{
${ }^{21}$ We suspect a sign error in their table in the case of one entry.

${ }^{22} \mathrm{We}$ also note that the ex post tax rate on income from capital varies so much that the mean of the distribution is not well estimated by the sample mean of a stochastic simulation as short as the ones reported in Table 2 of Chari et al. (1994). Simulations of our log-linear policy rules of only that length resulted in sample means for the ex post capital income tax rate ranging between +1 and -1 percent, even though we know on analytical grounds that the mean tax rate is zero, as reported in Table 3. Thus it is hard to tell, based on sample means from stochastic simulations as short as those reported by Chari et al., how much of a difference there really is between the moments implied by the approximation method used by Chari et al. and those implied by our LQ approximation.
} 
However, it may nonetheless be wondered how accurate such an approximation is in the case of disturbances of the size typically experienced by the U.S. economy. Indeed, Chari et al. (1994) adopt a minimum-weighted residual method for computing approximations to the optimal decision rules because they report having found that a "log-linearization method" led to a substantially less accurate characterization of the optimal dynamics of the tax rates (1995, pp. 383-390). This suggests that we should be concerned about the numerical accuracy of our log-linear approximation to the optimal tax rules.

As shown above, however, our results obtained using the LQ approximation (Table 3) are quite similar to those reported by Chari et al. (1994) on the basis of their nonlinear solution method. Of course, the sample moments reported in our table are not identical to theirs; but they are much more similar than are the results that Chari et al. (1995) report having obtained through "log-linearization". For example, in their Figure 12.3, Chari et al. (1995) report having obtained a higher standard deviation of the labor tax rate under the $\log$-linearization approach for all values of $\varphi$, and a vastly higher variance in the case of values of $\varphi$ near a critical value (between -3 and -4$)$ where the optimal variability of the labor tax rate falls essentially to zero according to their nonlinear computations, but remains high according to the log-linearization. But we also find with our LQ approximation ${ }^{23}$ that the optimal variability of the labor income tax falls nearly to zero for a critical value of $\varphi,{ }^{24}$ while it rises sharply for values of $\varphi$ on either side of these values (see Figure 1), just as in the figure of Chari et al.

Similarly, in their Figure 12.4A, they report that their log-linearization implies that the mean ex-ante capital tax rate should be positive for all values of $\varphi$, and substantially so (more than 3 percent) for large negative values of $\varphi$, while their nonlinear method indicates that the mean rate should be zero for the case $\varphi=0$, and slightly negative in the case of $\varphi<0$. But we find with our LQ approximation

\footnotetext{
${ }^{23}$ In considering the effects of variation in $\varphi$ in Figures 1 and 2, we assume a value of $\bar{\tau}^{h}$ as a function of $\varphi$ that linearly interpolates between the two values specified in Table 1 .

${ }^{24}$ In our computations, the critical value of $\varphi$ is between -4 and -5 , as is also reported in Chari et al. (1994, Figure 3, panel (a)). Note that we assign parameter values as in Chari et al. (1994), which uses a slightly different calibration than the one in Chari et al. (1995). Our Figure 1 still differs somewhat from Figure 3(a) in Chari et al. (1994), mainly because our assumption of a simple linear relation between $\bar{\tau}^{h}$ and $\varphi$ does not exactly capture the way in which the steady state varies with $\varphi$ in their simulations, as shown by Figure 2(a) of their paper.
} 
that the mean ex ante capital tax rate is zero for all values of $\varphi$, rather than being positive at all.

Finally, in their Figure 12.4B, they report that their log-linearization implies that the standard deviation of the ex ante capital tax rate remains above 2 percent for all $\varphi \leq 0$, while their nonlinear method shows that it falls to zero when $\varphi=0$. But we also find with our LQ approximation that the standard deviation falls to zero when $\varphi=0$ (see Figure 2). The LQ approximation proposed here clearly leads to quite different, and much more accurate, results than the log-linearization method employed by Chari et al. (1995). ${ }^{25}$

A measure of the accuracy of the LQ approximate solution that does not depend on comparison with the results of Chari et al. can be obtained by considering how closely the log-linear dynamics that solve the LQ problem come to satisfying the exact nonlinear first-order conditions that characterize optimal Ramsey policy (discussed above in section 1.4). Here we do not treat this issue in detail (as it is unclear how large a discrepancy should be regarded as acceptable), but consider, for purposes of illustration, the degree to which our log-linear dynamics fit the Euler equation for optimality of the rate of investment. ${ }^{26}$

As shown in the appendix, the stochastic version of Euler equation (1.34), after using (1.32) to substitute for the Lagrange multiplier $\lambda_{1 t}$, can be written in the form

$$
R_{t}=0
$$

where $R_{t}$ is the value of the left-hand side of (the stochastic version of) equation

\footnotetext{
${ }^{25}$ Chari et al. report that their log-linearization method yielded fairly accurate results regarding the optimal allocation of resources, but much less accurate results for the dynamics of the tax rates. They propose that this is because "the policies depend on ratios of the derivatives of the utility function and small errors in computing the allocations can lead to large errors in computing the policies" (1995, p. 383). We doubt that this is a correct explanation of their results. For example, given the optimal allocation, the optimal labor tax rate is given by solving (1.8), substituting (1.11) for the real wage. But the resulting mapping from allocations to the tax rate is exactly linear in $\tau_{t}^{h}, \hat{c}_{t}, \hat{k}_{t}$, and $\hat{z}_{t}$; it is only the non-linearity of the dependence on $\hat{h}_{t}$ that can possibly be a source of error in our log-linear approximation to this relation, and even the dependence on that variable is not more severely nonlinear than other equations involved in the determination of the optimal allocation. Judd (1996, sec. 4) suggests that the inaccurate results reported by Chari et al. result from their using an "ad hoc" method that does not result in a correct linear approximation to the optimal policy rules, unlike our corrected LQ method.

${ }^{26}$ This is also the sole optimality condition for which Chari et al. (1995) discuss the size of the Euler-equation residuals associated with their alternative numerical solutions.
} 
(1.34). In the case of the log-linear dynamics that solve the LQ problem, the (exact) value of the residual $R_{t}$ each period depends only on the state vector $v_{t}$ at that time; in particular, we can write

$$
R_{t}=R\left(v_{t}\right) \equiv m\left(v_{t}\right)-E\left[n\left(v_{t+1}\right) \mid v_{t}\right],
$$

where $v_{t}$ is the same state vector as in (3.8), and $m(v)$ and $n(v)$ are explicit, nonlinear functions defined in the appendix. The functions $m(v)$ and $n(v)$ can be computed given numerical values for the model parameters, and the steady-state values $\bar{h}$ and $\lambda_{0}$, that are computed in the manner explained earlier. The simulations provide sequences $\left\{v_{t}\right\}$ for the state variables, that allow $m\left(v_{t}\right)$ to be evaluated for each period. They also provide a value for $\hat{k}_{t+1}$ that (as a predetermined state variable) should be known with certainty as of period $t$. Since we also know, given the state $v_{t}$ in any period $t$, the probabilities of each of the finite number of possible values of $\hat{z}_{t+1}$ and $\hat{g}_{t+1}$ in the following period, we therefore have a complete conditional probability distribution for the possible values of $v_{t+1}$, allowing $E\left[n\left(v_{t+1}\right) \mid v_{t}\right]$ to be evaluated for each period of the simulation. This allows us to compute a residual sequence $\left\{R_{t}\right\}$ associated with the simulation; the size of these residuals provides a measure of the accuracy of the approximate policy rules.

Figure 3 shows the histograms of these residuals for simulations of the log-linear approximate policy rules obtained for each of the five cases in Table $3 .{ }^{27}$ One observes that the residuals are quite small. In the case of log utility (the baseline preference assumption) and serially correlated disturbances, the typical residual is on the order of -.0001, meaning that the current marginal utility of consumption is smaller than the level implied by the Euler equation by amount that is only .01 percent of the steadystate marginal utility of consumption. The residuals are an order of magnitude smaller in the case of serially uncorrelated disturbances (case 5), though they are three to four times as large in the high-risk-aversion case as in the baseline case. ${ }^{28}$

Another way of assessing the accuracy of the LQ approximation is comparing the results obtained using this approximation to those that would be obtained by simulating second-order Taylor series approximations to the optimal policy rules,

\footnotetext{
${ }^{27}$ The simulations are the same ones that generate the sample moments reported in Table 5 below.

${ }^{28} \mathrm{We}$ are unable to compare this degree of accuracy to that reported for the log-linearization approach used in Chari et al. (1995), as the units in which the residuals are reported in their figures are unclear.
} 
rather than the first-order approximations yielded by the LQ approximation. ${ }^{29} \mathrm{We}$ compute the coefficients of the second-order approximations to the optimal policy rules (i.e., to the solution to the nonlinear first-order conditions characterizing Ramsey policy) using the algorithm of Schmitt-Grohé and Uribe (2004), and then determine the implied moments of the dynamics of the tax rate through Monte Carlo simulation. In order to distinguish differences in the moments due to simulation (i.e., to sampling error) from differences due to the inclusion of second-order terms in the policy rules, we first present (in Table 5) the moments implied by the log-linearized policy rules, ${ }^{30}$ using Monte Carlo simulation to estimate the moments, rather than calculation of the exact moments as in Table $3 .^{31}$ (The numbers are similar, but not identical to the ones reported in Table 3, mainly as a result of sampling error.) Here we report the various statistics to three decimal places, rather than only two as in Table $3,{ }^{32}$ to allow the small differences that are made by a second-order approximation more evident.

Table 6 then displays the same moments, in the case that quadratic approximations to the optimal policy rules are used in the stochastic simulation, rather than the linear rules. (We use exactly the same sequences for the exogenous disturbances in the two cases, so that sampling error does not exaggerate the difference made by the second-order terms.) We find that the inclusion of second-order terms in the various equations used to derive our approximation to the optimal dynamics of the tax rates has only very small effects on the moments reported in Table 5. This is true despite

\footnotetext{
${ }^{29}$ Second-order perturbation methods of this kind have been widely advocated in the recent literature as a way of ensuring that welfare is correctly evaluated to second order in the amplitude of the disturbances; see, e.g., Jin and Judd (2002), Kim et al. (2003), and Schmitt-Grohé and Uribe (2004a, 2004b).

${ }^{30}$ Here the exact coefficients of the log-linear policy rules are also those computed using the algorithm of Schmitt-Grohé and Uribe, i.e., direct log-linearization of the nonlinear first-order conditions that characterize Ramsey policy, rather than solution of the LQ problem defined above. This alternative approach to the computation of the log-linearized policy rules results in small numerical differences from the policy rules used to generate the statistics reported in Table 3, though one can show analytically that the two approaches should yield identical coefficients.

${ }^{31}$ The statistics reported in both Tables 5 and 6 are based on stochastic simulation of the approximate optimal policy rules for 500,000 periods, with the first 60,000 periods of each simulation discarded to eliminate the effects of the arbitrary initial conditions.

${ }^{32}$ The degree of precision in Table 3 is chosen to match that of the results reported in Table 2 of Chari et al. (1994).
} 
the fact that the second-order corrections to the policy rules do substantially reduce the size of the Euler equation residuals, and thus do represent a better approximation to the true nonlinear optimal policy rules. ${ }^{33}$ This suggests that a log-linear approximation to the optimal policy functions is fairly accurate, in the case of disturbances of the size assumed in the exercise of Chari et al. (1994). ${ }^{34}$

\section{Conclusion}

We have shown, in the context of a familiar dynamic optimal taxation problem, that a naive approach to linear-quadratic approximation of the problem would yield results that are quite incorrect. At the same time, we have shown that it is possible to define an alternative quadratic objective - one that also corresponds to expected utility of the representative household, up to second order in the amplitude of the disturbances, in the case of any possible equilibrium, but that involves variables other than the arguments of the utility function itself - such that the LQ problem with this objective yields a correct local log-linear approximation to optimal policy in the case of small enough disturbances. We have also shown that the error involved in such a local log-linear approximation to the optimal policy appears not to be large, in the case of disturbances of the size that occur in a model calibrated to match features of U.S. time series. This suggests that LQ approximation methods of the kind illustrated here can usefully be employed in the analysis of optimal tax policy, both as a simple approach to computation and as a source of insight into the nature of optimal policy rules.

\footnotetext{
${ }^{33}$ The root-mean-square size of the Euler equation residuals corresponding to those shown in Figure 3 is reduced by a factor greater than 35 in the baseline case (case 1) when the second-order corrections are used; similarly dramatic reductions occur in the other cases. The Euler equation residuals are also now nearly zero on average, rather than being almost always negative as shown in Figure 3 .

${ }^{34}$ Schmitt-Grohé and Uribe (2004b) reach a similar conclusion in the context of a calibrated sticky-price model.
} 


\section{Appendix}

\section{Derivation of equation (2.1).}

Starting from (1.1), we note that a second-order Taylor series expansion of $u\left(c_{t}, h_{t}\right)$ takes the form

$$
\begin{aligned}
u\left(c_{t}, h_{t}\right)= & \bar{u}+\bar{u}_{c}\left(c_{t}-\bar{c}\right)+\bar{u}_{h}\left(h_{t}-\bar{h}\right)+\frac{1}{2} \bar{u}_{c c}\left(c_{t}-\bar{c}\right)^{2}+ \\
& +\frac{1}{2} \bar{u}_{h h}\left(h_{t}-\bar{h}\right)^{2}+\bar{u}_{c h}\left(c_{t}-\bar{c}\right)\left(h_{t}-\bar{h}\right)+\mathcal{O}\left(\|\xi\|^{3}\right),
\end{aligned}
$$

where partial derivatives are all evaluated at the steady state values $(\bar{c}, \bar{h})$ of the arguments of $u$.

We wish instead to expand in powers of the log deviations of consumption and hours from their steady-state levels. We note that in general,

$$
x_{t}-\bar{x}=\bar{x}\left(1+\hat{x}+\frac{\hat{x}^{2}}{2}\right)+\mathcal{O}\left(\|\xi\|^{3}\right),
$$

where $\hat{x} \equiv \ln x_{t} / \bar{x}$. Using this to substitute for $c_{t}-\bar{c}$ and $h_{t}-\bar{h}$ in the above expression, and suppressing terms of higher than second order in the log deviations, we obtain $(2.1)$.

\section{Derivation of equation (2.8).}

We begin by taking a second-order approximation of equation (1.15) in terms of $\log$ deviations from the steady-state values of the various endogenous variables. Rewriting the equation as

$$
y_{t}-\bar{y}=\left(c_{t}-\bar{c}\right)+\left(g_{t}-\bar{g}\right)+\left(k_{t+1}-\bar{k}\right)-(1-\tilde{\delta})\left(k_{t}-\bar{k}\right)
$$

we then obtain

$\hat{y}_{t}=s_{c} \hat{c}_{t}+s_{g} \hat{g}_{t}+s_{k}\left(\hat{k}_{t+1}-(1-\tilde{\delta}) \hat{k}_{t}\right)+\frac{1}{2} s_{c} \hat{c}_{t}^{2}+\frac{1}{2} s_{g} \hat{g}_{t}^{2}+\frac{1}{2} s_{k}\left(\hat{k}_{t+1}^{2}-(1-\tilde{\delta}) \hat{k}_{t}^{2}\right)-\frac{1}{2} \hat{y}_{t}^{2}+\mathcal{O}\left(\|\xi\|^{3}\right)$,

where $\hat{g}_{t} \equiv \log \left(g_{t} / \bar{g}\right)$ and $s_{c} \equiv \bar{c} / \bar{y}, s_{g} \equiv \bar{g} / \bar{y}$, and $s_{k} \equiv \bar{k} / \bar{y}$.

We similarly take a second-order approximation to the production function $y_{t}=$ $f\left(k_{t}, z_{t} h_{t}\right)$, which can be written as

$$
\begin{aligned}
y_{t}-\bar{y}= & \bar{f}_{k}\left(k_{t}-\bar{k}\right)+\bar{z} \bar{f}_{h}\left(h_{t}-\bar{h}\right)+\bar{h} \bar{f}_{h}\left(z_{t}-\bar{z}\right)+\frac{1}{2} f_{k k}\left(k_{t}-\bar{k}\right)^{2}+\frac{1}{2} \bar{z}^{2} \bar{f}_{h h}\left(h_{t}-\bar{h}\right)^{2}+ \\
& +\bar{z} f_{k h}\left(k_{t}-\bar{k}\right)\left(h_{t}-\bar{h}\right)+\bar{h} \bar{f}_{k h}\left(k_{t}-\bar{k}\right)\left(z_{t}-\bar{z}\right)+\bar{f}_{h}\left(h_{t}-\bar{h}\right)\left(z_{t}-\bar{z}\right) \\
& +\bar{h} \bar{z} \bar{f}_{h h}\left(h_{t}-\bar{h}\right)\left(z_{t}-\bar{z}\right)+\text { s.o.t.i.p. }+\mathcal{O}\left(\|\xi\|^{3}\right) .
\end{aligned}
$$


This can be expressed in terms of log deviations as

$$
\begin{aligned}
\hat{y}_{t}+\frac{1}{2} \hat{y}_{t}^{2}= & \alpha\left(\hat{k}_{t}+\frac{1}{2} \hat{k}_{t}^{2}\right)+(1-\alpha)\left(\hat{h}_{t}+\hat{z}_{t}+\frac{1}{2} \hat{h}_{t}^{2}\right)+(1-\alpha) \hat{h}_{t} \hat{z}_{t}-\frac{1}{2} \alpha(1-\alpha) \hat{k}_{t}^{2}+ \\
& +\frac{1}{2} \alpha(1-\alpha) \hat{h}_{t}^{2}-\alpha(1-\alpha) \hat{k}_{t} \hat{h}_{t}+\alpha(1-\alpha) \hat{k}_{t} \hat{z}_{t}-\alpha(1-\alpha) \hat{h}_{t} \hat{z}_{t}+\text { s.o.t.i.p. }+\mathcal{O}\left(\|\xi\|^{3}\right),
\end{aligned}
$$

which can be finally written as

$$
\begin{aligned}
\hat{y}_{t}+\frac{1}{2} \hat{y}_{t}^{2}= & \alpha\left(\hat{k}_{t}+\frac{1}{2} \hat{k}_{t}^{2}\right)+(1-\alpha)\left(\hat{h}_{t}+\hat{z}_{t}+\frac{1}{2} \hat{h}_{t}^{2}\right)+(1-\alpha) \hat{h}_{t} \hat{z}_{t}+ \\
& -\frac{1}{2} \alpha(1-\alpha)\left(\hat{k}_{t}-\hat{z}_{t}-\hat{h}_{t}\right)^{2}+\text { s.o.t.i.p. }+\mathcal{O}\left(\|\xi\|^{3}\right) .
\end{aligned}
$$

We can substitute in (4.1) to obtain

$$
\begin{aligned}
0= & s_{c} \hat{c}_{t}-(1-\alpha)\left(\hat{z}_{t}+\hat{h}_{t}\right)+s_{g} \hat{g}_{t}+s_{k}\left(\hat{k}_{t+1}-(1-\tilde{\delta}) \hat{k}_{t}\right)-\alpha \hat{k}_{t}+\frac{1}{2} s_{c} \hat{c}_{t}^{2}+\frac{1}{2} s_{k}\left(\hat{k}_{t+1}^{2}-(1-\tilde{\delta}) \hat{k}_{t}^{2}\right) \\
& -\frac{1}{2} \alpha \hat{k}_{t}^{2}+\frac{1}{2} \alpha(1-\alpha) \tilde{k}_{t}^{2}-\frac{1}{2}(1-\alpha)\left(\hat{h}_{t}^{2}+2 \hat{z}_{t} \hat{h}_{t}\right)+\text { s.o.t.i.p. }+\mathcal{O}\left(\|\xi\|^{3}\right) .
\end{aligned}
$$

Integrating forward, we finally obtain

$0=\sum_{t=t_{0}}^{\infty} \tilde{\beta}^{t-t_{0}}\left\{s_{c}\left(\hat{c}_{t}+\frac{1}{2} \hat{c}_{t}^{2}\right)-(1-\alpha)\left(\hat{h}_{t}+\frac{1}{2} \hat{h}_{t}^{2}+\hat{h}_{t} \hat{z}_{t}\right)+\frac{1}{2} \alpha(1-\alpha) \hat{\tilde{k}}_{t}^{2}\right\}+$ t.i.p. $+\mathcal{O}\left(\|\xi\|^{3}\right)$.

\section{Derivation of equation (2.10)}

Starting from (1.19), we first note that

$$
\begin{aligned}
u_{c}\left(c_{t}, h_{t}\right) c_{t}+u_{h}\left(c_{t}, h_{t}\right) h_{t}= & \bar{u}_{c} \bar{c}+\bar{u}_{h} \bar{h}+\bar{u}_{c}\left(c_{t}-\bar{c}\right)+ \\
& \bar{c}\left[\bar{u}_{c c}\left(c_{t}-\bar{c}\right)+\bar{u}_{c h}\left(h_{t}-\bar{h}\right)+\frac{1}{2} \bar{u}_{c c c}\left(c_{t}-\bar{c}\right)^{2}+\right. \\
& \left.\frac{1}{2} \bar{u}_{c h h}\left(h_{t}-\bar{h}\right)^{2}+\bar{u}_{c c h}\left(c_{t}-\bar{c}\right)\left(h_{t}-\bar{h}\right)\right]+\bar{u}_{c c}\left(c_{t}-\bar{c}\right)^{2} \\
& +\bar{u}_{c h}\left(c_{t}-\bar{c}\right)\left(h_{t}-\bar{h}\right)+\bar{u}_{h}\left(h_{t}-\bar{h}\right)+ \\
& +\bar{h}\left[\bar{u}_{h c}\left(c_{t}-\bar{c}\right)+\bar{u}_{h h}\left(h_{t}-\bar{h}\right)+\frac{1}{2} \bar{u}_{h h h}\left(h_{t}-\bar{h}\right)^{2}+\right. \\
& \left.+\frac{1}{2} \bar{u}_{h c c}\left(c_{t}-\bar{c}\right)^{2}+\bar{u}_{h h c}\left(h_{t}-\bar{h}\right)\left(c_{t}-\bar{c}\right)\right]+ \\
& \bar{u}_{h h}\left(h_{t}-\bar{h}\right)^{2}+\bar{u}_{h c}\left(h_{t}-\bar{h}\right)\left(c_{t}-\bar{c}\right)+\mathcal{O}\left(\|\xi\|^{3}\right)
\end{aligned}
$$


and then obtain

$$
\begin{aligned}
u_{c}\left(c_{t}, h_{t}\right) c_{t}+u_{h}\left(c_{t}, h_{t}\right) h_{t}= & \bar{u}_{c} \bar{c}\left[\hat{c}_{t}+\frac{1}{2} \hat{c}_{t}^{2}\right]+\bar{c}\left[\bar{u}_{c c} \bar{c}\left(\hat{c}_{t}+\frac{1}{2} \hat{c}_{t}^{2}\right)+\bar{u}_{c h} \bar{h}\left(\hat{h}_{t}+\frac{\hat{h}_{t}^{2}}{2}\right)\right. \\
& \left.+\frac{1}{2} \bar{u}_{c c c} \bar{c}^{2} \hat{c}_{t}^{2}+\frac{1}{2} \bar{u}_{c h h} \bar{h}^{2} \hat{h}_{t}^{2}+\bar{u}_{c c h} \bar{c} \bar{h} \hat{c}_{t} \hat{h}_{t}\right]+\bar{u}_{h} \bar{h}\left(\hat{h}_{t}+\frac{\hat{h}_{t}^{2}}{2}\right)+ \\
& +\bar{u}_{c c} \bar{c}^{2} \hat{c}_{t}^{2}+\bar{u}_{c h} \bar{c} \bar{h} \hat{c}_{t} \hat{h}_{t} \\
& +\bar{h}\left[\bar{u}_{h c} \bar{c}\left(\hat{c}_{t}+\frac{1}{2} \hat{c}_{t}^{2}\right)+\bar{u}_{h h} \bar{h}\left(\hat{h}_{t}+\frac{\hat{h}_{t}^{2}}{2}\right)+\frac{1}{2} \bar{u}_{h h h} \bar{h}^{2} \hat{h}_{t}^{2}+\right. \\
& \left.+\frac{1}{2} \bar{u}_{h c c} \bar{c}^{2} \hat{c}_{t}^{2}+\bar{u}_{h h c} \bar{c} \hat{c}_{t} \hat{h}_{t}\right]+\bar{u}_{h h} \bar{h}^{2} \hat{h}_{t}^{2}+ \\
& +\bar{u}_{c h} \bar{c} \bar{h} \hat{c}_{t} \hat{h}_{t}+\text { t.i.p. }+\mathcal{O}\left(\|\xi\|^{3}\right) .
\end{aligned}
$$

We can simplify the above expression to

$$
\begin{aligned}
u_{c}\left(c_{t}, h_{t}\right) c_{t}+u_{h}\left(c_{t}, h_{t}\right) h_{t}= & \bar{u}_{c} \bar{c}\left\{\left(1-\sigma^{-1}\right)\left(\hat{c}_{t}+\frac{1}{2} \hat{c}_{t}^{2}\right)+\psi\left(\hat{h}_{t}+\frac{\hat{h}_{t}^{2}}{2}\right)\right. \\
& -\frac{1}{2} \sigma^{-1} \sigma_{1}^{-1} \hat{c}_{t}^{2}+\frac{1}{2} \psi \psi_{1} \hat{h}_{t}^{2}-\sigma^{-1} \psi_{2} \hat{c}_{t} \hat{h}_{t}-\phi\left(\hat{h}_{t}+\frac{\hat{h}_{t}^{2}}{2}\right)+ \\
& -\sigma^{-1} \hat{c}_{t}^{2}+\psi \hat{h}_{t} \hat{c}_{t}+\psi\left(\hat{c}_{t}+\frac{1}{2} \hat{c}_{t}^{2}\right) \\
& -\phi \nu\left(\hat{h}_{t}+\frac{\hat{h}_{t}^{2}}{2}\right)-\frac{1}{2} \phi \nu \nu_{1} \hat{h}_{t}^{2}-\frac{1}{2} \sigma^{-1} \psi_{2} \hat{c}_{t}^{2} \\
& \left.+\psi \psi_{1} \hat{c}_{t} \hat{h}_{t}-\phi \nu \hat{h}_{t}^{2}+\psi \hat{h}_{t} \hat{c}_{t}\right\}+ \text { t.i.p. }+\mathcal{O}\left(\|\xi\|^{3}\right),
\end{aligned}
$$

where $\sigma_{1}^{-1} \equiv \bar{u}_{c c c} \bar{c} / \bar{u}_{c c}, \psi_{1} \equiv \bar{u}_{c h h} \bar{h} / \bar{u}_{c h}, \psi_{2} \equiv \bar{u}_{c c h} \bar{h} / \bar{u}_{c c}, \nu_{1} \equiv \bar{u}_{h h h} \bar{h} / \bar{u}_{h h}$. Plugging the above equation into (1.19), we obtain that

$$
\begin{aligned}
\frac{W_{t_{0}}-\bar{W}}{\bar{u}_{c} \bar{c}}= & E_{t_{0}} \sum_{t=t_{0}}^{\infty} \tilde{\beta}^{t-t_{0}}\left\{\left(1-\sigma^{-1}+\psi\right) \hat{c}_{t}+(\psi-\phi-\phi \nu) \hat{h}_{t}\right. \\
& +\frac{1}{2}\left(\psi+\psi \psi_{1}-\phi-\phi \nu-\phi \nu \nu_{1}-2 \phi \nu\right) \hat{h}_{t}^{2}+ \\
& +\frac{1}{2}\left(1-\sigma^{-1}+\psi-\sigma^{-1} \sigma_{1}^{-1}-\sigma^{-1} \psi_{2}-2 \sigma^{-1}\right) \hat{c}_{t}^{2}+ \\
& \left.+\left(\psi \psi_{1}-\sigma^{-1} \psi_{2}+2 \psi\right) \hat{c}_{t} \hat{h}_{t}\right\} \\
& + \text { s.o.t.i.p. }+\mathcal{O}\left(\|\xi\|^{3}\right) .
\end{aligned}
$$

We further note that

$$
W_{t} \equiv b_{t-1}^{s} u_{c}\left(c_{t}, h_{t}\right)+u_{c}\left(c_{t}, h_{t}\right) k_{t}\left[(1-\tilde{\delta})+\left(1-\tau_{t}^{k}\right) f_{k}\left(z_{t} h_{t}, k_{t}\right)+\delta \rho^{-1} \tau_{t}^{k}\right],
$$


where

$$
\delta \rho^{-1}=\rho^{-1}-(1-\tilde{\delta}) .
$$

We then have as a first-order expansion of the constraint that

$$
\begin{aligned}
W_{t}-\bar{W} \equiv & \bar{u}_{c}\left(b_{t-1}-\bar{b}\right)+\bar{b}\left[\bar{u}_{c c}\left(c_{t}-\bar{c}\right)+\bar{u}_{c h}\left(h_{t}-\bar{h}\right)\right]+ \\
& +\frac{1}{\tilde{\beta}}\left[\bar{u}_{c}\left(k_{t}-\bar{k}\right)+\bar{k} \bar{u}_{c c}\left(c_{t}-\bar{c}\right)+\bar{k} \bar{u}_{c h}\left(h_{t}-\bar{h}\right)\right]+ \\
& +\bar{u}_{c} \bar{k}\left\{\left[\bar{f}_{k k}\left(k_{t}-\bar{k}\right)+\bar{h} \bar{f}_{k h}\left(z_{t}-\bar{z}\right)+\bar{z} \bar{f}_{k h}\left(h_{t}-\bar{h}\right)\right]-\left(\bar{f}_{k}-\delta \rho^{-1}\right) \tau_{t}^{k}\right\}+\mathcal{O}\left(\|\xi\|^{2}\right),
\end{aligned}
$$

which can be written as

$$
\begin{aligned}
W_{t}-\bar{W}= & \bar{u}_{c} \bar{b} \hat{b}_{t-1}-\bar{u}_{c} \bar{b}\left[\sigma^{-1} \hat{c}_{t}-\psi \hat{h}_{t}\right]+\frac{\bar{u}_{c} \bar{k}}{\tilde{\beta}}\left(\hat{k}_{t}-\sigma^{-1} \hat{c}_{t}+\psi \hat{h}_{t}\right)+ \\
& +\bar{u}_{c} \bar{k}^{2} \bar{f}_{k k}\left(\hat{k}_{t}-\hat{z}_{t}-\hat{h}_{t}\right)-\bar{u}_{c} \bar{k}\left(\bar{f}_{k}-\delta \rho^{-1}\right) \tau_{t}^{k}+\mathcal{O}\left(\|\xi\|^{2}\right) .
\end{aligned}
$$

Hence

$$
\begin{aligned}
s_{c} \tilde{W}_{t}= & s_{b} \hat{b}_{t-1}-s_{b}\left[\sigma^{-1} \hat{c}_{t}-\psi \hat{h}_{t}\right]+\tilde{\beta}^{-1} s_{k}\left(\hat{k}_{t}-\sigma^{-1} \hat{c}_{t}+\psi \hat{h}_{t}\right) \\
& -\alpha(1-\alpha)\left(\hat{k}_{t}-\hat{z}_{t}-\hat{h}_{t}\right)-\left(\alpha-s_{k} \delta \rho^{-1}\right) \tau_{t}^{k}+\mathcal{O}\left(\|\xi\|^{2}\right),
\end{aligned}
$$

where $s_{b} \equiv \bar{b} / \bar{y}$ and $\tilde{W}_{t} \equiv\left(\tilde{W}_{t}-\bar{W}\right) / \bar{u}_{c} \bar{c}$.

We finally note that

$$
\left(\alpha-s_{k} \delta \rho^{-1}\right)=s_{k}\left(\alpha s_{k}^{-1}-\rho^{-1}+(1-\tilde{\delta})\right)=s_{k}\left(\tilde{\beta}^{-1}-\rho^{-1}\right) .
$$

We have then that

$$
\begin{gathered}
\tilde{W}_{t}=E_{t} \sum_{T=t}^{\infty} \tilde{\beta}^{T-t_{0}}\left\{d_{c} \hat{c}_{T}+d_{h} \hat{h}_{T}\right\} \\
s_{c} \tilde{W}_{t}=s_{b} \hat{b}_{t-1}^{s}-b_{c} \hat{c}_{t}+b_{h} \hat{h}_{t}+b_{k} \hat{k}_{t}+s_{c}^{-1} \alpha(1-\alpha) \hat{z}_{t}-b_{\tau} \tau_{t}^{k},
\end{gathered}
$$

where we have defined $b_{c} \equiv s_{b} \sigma^{-1}+\tilde{\beta}^{-1} s_{k} \sigma^{-1}, b_{h} \equiv s_{b} \psi+\tilde{\beta}^{-1} s_{k} \psi+\alpha(1-\alpha)$, $b_{k} \equiv \tilde{\beta}^{-1} s_{k}-\alpha(1-\alpha), d_{c} \equiv\left(1-\sigma^{-1}+\psi\right), d_{h} \equiv(\psi-\phi-\phi \nu)$ and $b_{\tau} \equiv s_{k}\left(\tilde{\beta}^{-1}-\rho^{-1}\right)$.

\section{Second-order conditions}

The optimal solution to the linear-quadratic problem minimize the following loss function

$$
L_{t_{0}}=\frac{1}{2} E_{t_{0}} \sum_{t=t_{0}}^{\infty} \tilde{\beta}^{t-t_{0}}\left\{q_{c}\left(\hat{c}_{t}-\theta \hat{h}_{t}\right)^{2}+q_{h}\left(\hat{h}_{t}-h_{t}^{*}\right)^{2}+q_{k}\left[\hat{k}_{t}-\left(\hat{z}_{t}+\hat{h}_{t}\right)\right]^{2}\right\}
$$


under the constraint

$$
\hat{k}_{t+1}=\tilde{\beta}^{-1} \hat{k}_{t}-s_{k}^{-1} s_{c} \hat{c}_{t}+s_{k}^{-1}\left(1-\alpha_{k}\right)\left(\hat{z}_{t}+\hat{h}_{t}\right)-s_{k}^{-1} s_{g} \hat{g}_{t}
$$

for each $t \geq t_{0}$ and the constraint

$$
\tilde{W}_{t_{0}}=E_{t_{0}} \sum_{T=t_{0}}^{\infty} \tilde{\beta}^{T-t_{0}}\left\{d_{c} \hat{c}_{T}+d_{h} \hat{h}_{T}\right\}
$$

at time $t_{0}$ given the initial conditions $\hat{k}_{t_{0}}$ and $\tilde{W}_{t_{0}}=\bar{W}_{t_{0}}$.

We study under which conditions the solution to the above optimization problem corresponds indeed to a minimum, i.e. under which conditions second order conditions are satisfied. This analysis boils down to study under which restrictions the quadratic form (4.3) is positive definite under the sequence of constraints (4.4) and the constraint (4.5). Let us assume that $\left\{\hat{c}_{t}, \hat{h}_{t}, \hat{k}_{t+1}\right\}_{t=t_{0}}^{\infty}$ is an optimal plan for the above problem and define the sequences

$$
\begin{aligned}
\hat{c}_{t}^{\dagger} & =\hat{c}_{t}+\psi_{c, t}, \\
\hat{h}_{t}^{\dagger} & =\hat{h}_{t}+\psi_{h, t}, \\
\hat{k}_{t+1}^{\dagger} & =\hat{k}_{t+1}+\psi_{k, t}
\end{aligned}
$$

where the processes $\left\{\psi_{c, t}, \psi_{h, t}, \psi_{k, t}\right\}_{t=t_{0}}^{\infty}$ are real valued and satisfy

$$
E_{t_{0}} \sum_{T=t_{0}}^{\infty} \tilde{\beta}^{T-t_{0}} \psi_{j, t}^{2}<\infty \quad \text { for } j=c, h, k,
$$

plus the sequence of constraints

$$
\psi_{k, t}=\tilde{\beta}^{-1} \psi_{k, t-1}-s_{k}^{-1} s_{c} \psi_{c, t}+s_{k}^{-1}(1-\alpha) \psi_{h, t}
$$

for each $t \geq t_{0}$ and

$$
E_{t_{0}} \sum_{T=t_{0}}^{\infty} \tilde{\beta}^{T-t_{0}}\left\{d_{c} \psi_{c, T}+d_{h} \psi_{h, T}\right\}=0
$$

at time $t_{0}$ with initial condition $\psi_{k, t_{0}-1}=0$. It follows that the process $\left\{\hat{c}_{t}^{\dagger}, \hat{h}_{t}^{\dagger}\right.$, $\left.\hat{k}_{t+1}^{\dagger}\right\}_{t=t_{0}}^{\infty}$ is a feasible perturbation to the optimal plan and achieves a loss given by

$$
\begin{aligned}
L_{t_{0}}\left(\hat{c}^{\dagger}, \hat{h}^{\dagger}, \hat{k}^{\dagger}\right)= & L_{t_{0}}(\hat{c}, \hat{h}, \hat{k})+E_{t_{0}} \sum_{t=t_{0}}^{\infty} \tilde{\beta}^{t-t_{0}}\left[q_{c}\left(\hat{c}_{t}-\theta \hat{h}_{t}\right)\left(\psi_{c, t}-\theta \psi_{h, t}\right)+q_{h} \psi_{h, t}\left(\hat{h}_{t}-h_{t}^{*}\right)+\right. \\
& +q_{k}\left(\left(\hat{k}_{t}-\left(\hat{z}_{t}+\hat{h}_{t}\right)\right)\left(\psi_{k, t-1}-\psi_{h, t}\right)\right]+ \\
& +E_{t_{0}} \sum_{t=t_{0}}^{\infty} \tilde{\beta}^{t-t_{0}}\left[q_{c}\left(\psi_{c, t}-\theta \psi_{h, t}\right)^{2}+q_{h} \psi_{h, t}^{2}+q_{k}\left(\psi_{k, t-1}-\psi_{h, t}\right)^{2}\right] .
\end{aligned}
$$


Since the sequences $\left\{\hat{c}_{t}, \hat{h}_{t}, \hat{k}_{t+1}\right\}_{t=t_{0}}^{\infty}$ satisfy necessary conditions for an optimal plan, then the second term on the RHS of the above equation is zero, i.e.

$E_{t_{0}} \sum_{t=t_{0}}^{\infty} \tilde{\beta}^{t-t_{0}}\left[q_{c}\left(\hat{c}_{t}-\theta \hat{h}_{t}\right)\left(\psi_{c, t}-\theta \psi_{h, t}\right)+q_{h} \psi_{h, t}\left(\hat{h}_{t}-h_{t}^{*}\right)+q_{k}\left(\left(\hat{k}_{t}-\left(\hat{z}_{t}+\hat{h}_{t}\right)\right)\left(\psi_{k, t-1}-\psi_{h, t}\right)\right]=0\right.$

It follows that the sequences $\left\{\hat{c}_{t}, \hat{h}_{t}, \hat{k}_{t+1}\right\}_{t=t_{0}}^{\infty}$ will be a minimum if and only

$$
E_{t_{0}} \sum_{t=t_{0}}^{\infty} \tilde{\beta}^{t-t_{0}}\left[q_{c}\left(\psi_{c, t}-\theta \psi_{h, t}\right)^{2}+q_{h} \psi_{h, t}^{2}+q_{k}\left(\psi_{k, t-1}-\psi_{h, t}\right)^{2}\right]>0
$$

for all processes $\left\{\psi_{c, t}, \psi_{h, t}, \psi_{k, t}\right\}_{t=t_{0}}^{\infty}$ satisfying (4.6), (4.7) and (4.8) given $\psi_{k, t_{0}-1}=0$.

First we show that we can disregard constraint (4.8) from the analysis. Let us define $\psi_{t} \equiv\left(\psi_{c, t}, \psi_{h, t}, \psi_{k, t}\right)$. Given a process $\left\{\psi_{t}\right\}_{t=t_{0}}^{\infty}$ that satisfies only the constraints (4.6) and (4.7) given $\psi_{k, t_{0}-1}=0$ and such that (4.9) is negative, we can construct a process $\left\{\bar{\psi}_{t}\right\}_{t=t_{0}}^{\infty}$ with $\bar{\psi}_{t_{0}}=0$ and $\bar{\psi}_{t+1}=\sigma_{t_{0}+1} \psi_{t}$ for each $t>t_{0}$, where $\sigma_{t_{0}+1}$ is a sunspot variable known at time $t_{0}+1$ which takes either value 1 or -1 with probability $1 / 2$. The constructed process $\left\{\bar{\psi}_{t}\right\}_{t=t_{0}}^{\infty}$ still satisfies (4.6), (4.7) and in addition it satisfies the constraint (4.8) while achieving a negative value for the loss function (4.9).

It follows that $\left\{\hat{c}_{t}, \hat{h}_{t}, \hat{k}_{t+1}\right\}_{t=t_{0}}^{\infty}$ will be a minimum if and only (4.9) is positive for all processes $\left\{\psi_{t}\right\}_{t=t_{0}}^{\infty}$ that satisfy only the constraints (4.6), (4.7) and $\psi_{k, t_{0}-1}=0$. In Benigno and Woodford (2004b), we further show that this stochastic problem can be reconnected to a deterministic problem of the kind analyzed by Telser and Graves (1972), in which the sequence $\left\{\psi_{t}\right\}_{t=t_{0}}^{\infty}$ can be complex valued.

To make this parallel, we can write the problem in a more compact way by rewriting the deterministic version of (4.9) as

$$
\sum_{t=0}^{\infty} \tilde{\beta}^{t} \tilde{x}_{t}^{\ddagger} B(L) \tilde{x}_{t}
$$

where we have defined

$$
\tilde{x}_{t}=\left[\begin{array}{c}
\psi_{x, t} \\
\psi_{h, t} \\
\psi_{k, t}
\end{array}\right]
$$


with $\psi_{x, t} \equiv \psi_{c, t}-\theta \psi_{h, t}$ and the matrix $B(L)$ as

$$
B(L)=\left[\begin{array}{ccc}
q_{c} & 0 & 0 \\
0 & q_{h}+q_{k} & -2 q_{k} L \\
0 & 0 & q_{k}
\end{array}\right] .
$$

Here $\tilde{x}_{t}^{\ddagger}$ denotes the conjugate transpose of $\tilde{x}_{t}$. We can also write the constraint (4.7) as

$$
A(L) \tilde{x}_{t}=0
$$

where

$$
A(L)=\left[\begin{array}{lll}
s_{k}^{-1} s_{c} & s_{k}^{-1} s_{c} \theta-s_{k}^{-1}(1-\alpha) & 1
\end{array}\right]+\left[\begin{array}{lll}
0 & 0 & -\tilde{\beta}^{-1}
\end{array}\right] L .
$$

The process $\left\{x_{t}\right\}$ now satisfies a bound of the form

$$
E_{t_{0}} \sum_{T=t_{0}}^{\infty} \tilde{\beta}^{T-t_{0}} \tilde{x}_{t}^{\ddagger} \tilde{x}_{t}<\infty
$$

Following Telser and Graves (1972), we can make the following transformation of variables by defining $x_{t}$ as $x_{t} \equiv \tilde{\beta}^{\frac{t}{2}} \tilde{x}_{t}$. Second-order conditions of the original problem are satisfied if and only if the following quadratic objective function

$$
\frac{1}{2} \sum_{t=0}^{\infty} x_{t}^{\ddagger} B\left(\tilde{\beta}^{\frac{1}{2}} L\right) x_{t}
$$

is positive definite for sequences $\left\{x_{t}\right\}$ that satisfies (4.10) and the sequence of constraints $A\left(\tilde{\beta}^{\frac{1}{2}} L\right) x_{t}=0$ at each $t \geq 0$ given $\psi_{k,-1}=0$.

Since the harmonic matrix

$$
\frac{1}{2}\left[B\left(\tilde{\beta}^{\frac{1}{2}} e^{-i \vartheta}\right)+B^{\prime}\left(\tilde{\beta}^{\frac{1}{2}} e^{i \vartheta}\right)\right]=\left[\begin{array}{ccc}
q_{c} & 0 & 0 \\
0 & q_{h}+q_{k} & -\tilde{\beta}^{\frac{1}{2}} q_{k} e^{-i \vartheta} \\
0 & -\tilde{\beta}^{\frac{1}{2}} q_{k} e^{i \vartheta} & \tilde{\beta} q_{k}
\end{array}\right]
$$

is non-singular for all $-\pi \leq \vartheta \leq \pi$ provided $q_{c}, q_{h}, q_{k}$ are all different from zero and since the matrix $A\left(\tilde{\beta}^{\frac{1}{2}} e^{-i \vartheta}\right)$ is of rank 1 for all $-\pi \leq \vartheta \leq \pi$, we can then use theorem 5.2 in Telser and Graves (1972) which allow to study second-order conditions in terms of the determinants of the following bordered Hessian

$$
H(\vartheta)=\left[\begin{array}{cc}
0 & A\left(\tilde{\beta}^{\frac{1}{2}} e^{-i \vartheta}\right) \\
A^{\prime}\left(\tilde{\beta}^{\frac{1}{2}} e^{i \vartheta}\right) & \frac{1}{2}\left(B\left(\tilde{\beta}^{\frac{1}{2}} e^{-i \vartheta}\right)+B^{\prime}\left(\tilde{\beta}^{\frac{1}{2}} e^{i \vartheta}\right)\right)
\end{array}\right]
$$


where in our case

$$
H(\vartheta)=\left[\begin{array}{cccc}
0 & s_{k}^{-1} s_{c} & s_{k}^{-1} s_{c} \theta-s_{k}^{-1}(1-\alpha) & 1-\tilde{\beta}^{\frac{1}{2}} e^{-i \vartheta} \\
s_{k}^{-1} s_{c} & q_{c} & 0 & 0 \\
s_{k}^{-1} s_{c} \theta-s_{k}^{-1}(1-\alpha) & 0 & q_{h}+q_{k} & -\tilde{\beta}^{\frac{1}{2}} q_{k} e^{-i \vartheta} \\
1-\tilde{\beta}^{\frac{1}{2}} e^{i \vartheta} & 0 & -\tilde{\beta}^{\frac{1}{2}} q_{k} e^{i \vartheta} & \tilde{\beta} q_{k}
\end{array}\right] .
$$

Since $m=1$, the second-order conditions are satisfied if and only the northwest principal minors of order $p>2$ have all the same sign as $(-1)^{m}=-1$. For the case $p=3$, we are interested in the matrix

$$
H_{1}=\left[\begin{array}{ccc}
0 & s_{k}^{-1} s_{c} & s_{k}^{-1} s_{c} \theta-s_{k}^{-1}(1-\alpha) \\
s_{k}^{-1} s_{c} & q_{c} & 0 \\
s_{k}^{-1} s_{c} \theta-s_{k}^{-1}(1-\alpha) & 0 & q_{h}+q_{k}
\end{array}\right] .
$$

The determinant of $H_{1}$ is negative if and only if (i) the inequality

$$
s_{c}^{2}\left(q_{h}+q_{k}\right)+q_{c}\left[(1-\alpha)-\theta s_{c}\right]^{2}>0
$$

is satisfied.

For the case $p=4$, we must also require the determinant of the bordered Hessian $H(\vartheta)$ to be negative for all $-\pi \leq \vartheta \leq \pi$. This determinant is equal to

$$
\begin{aligned}
\operatorname{det} H(\vartheta)= & -\tilde{\beta} s_{k}^{-2} s_{c}^{2} q_{k} q_{h}+q_{c}\left\{-s_{k}^{-1}\left[s_{c} \theta-(1-\alpha)\right]\left[s_{k}^{-1}\left(s_{c} \theta-(1-\alpha)\right) \tilde{\beta} q_{k}\right.\right. \\
& \left.\left.+q_{k} \tilde{\beta}^{\frac{1}{2}} e^{-i \vartheta}\left(1-\tilde{\beta}^{\frac{1}{2}} e^{i \vartheta}\right)\right]\right\}+q_{c}\left\{( 1 - \tilde { \beta } ^ { \frac { 1 } { 2 } } e ^ { - i \vartheta } ) \left[-\tilde{\beta}^{\frac{1}{2}} q_{k} e^{i \vartheta} s_{k}^{-1}\left(s_{c} \theta-(1-\alpha)\right)+\right.\right. \\
& \left.-\left(1-\tilde{\beta}^{\frac{1}{2}} e^{i \vartheta}\right)\left(q_{h}+q_{k}\right)\right] \\
= & -\tilde{\beta} s_{k}^{-2} s_{c}^{2} q_{k} q_{h}-\tilde{\beta} s_{k}^{-2} q_{k} q_{c}\left[(1-\alpha)-\theta s_{c}\right]^{2}-s_{k}^{-1} q_{c} q_{k}\left[s_{c} \theta-(1-\alpha)\right] \tilde{\beta}^{\frac{1}{2}} e^{-i \vartheta}\left(1-\tilde{\beta}^{\frac{1}{2}} e^{i \vartheta}\right) \\
& -s_{k}^{-1} q_{c} q_{k}\left[s_{c} \theta-(1-\alpha)\right] \tilde{\beta}^{\frac{1}{2}} e^{i \vartheta}\left(1-\tilde{\beta}^{\frac{1}{2}} e^{-i \vartheta}\right)-q_{c}\left(q_{h}+q_{k}\right)\left(1-\tilde{\beta}^{\frac{1}{2}} e^{i \vartheta}\right)\left(1-\tilde{\beta}^{\frac{1}{2}} e^{-i \vartheta}\right) \\
= & -\tilde{\beta} s_{k}^{-2} s_{c}^{2} q_{k} q_{h}-\tilde{\beta} s_{k}^{-2} q_{k} q_{c}\left[(1-\alpha)-\theta s_{c}\right]^{2}+\tilde{\beta}^{\frac{1}{2}}\left(2 \tilde{\beta}^{\frac{1}{2}}-2 \cos \vartheta\right)\left[(1-\alpha)-\theta s_{c}\right] s_{k}^{-1} q_{c} q_{k} \\
& -q_{c}\left(q_{h}+q_{k}\right)\left[1+\tilde{\beta}-2 \cos \vartheta \tilde{\beta}^{\frac{1}{2}}\right]
\end{aligned}
$$

It follows that

$$
\operatorname{det} H(\vartheta)<0 \text { for all }-\pi \leq \vartheta \leq \pi
$$

if and only if $(i i)$ the inequality

$$
\begin{aligned}
& \tilde{\beta} s_{k}^{-2} s_{c}^{2} q_{k} q_{h}+\tilde{\beta} s_{k}^{-2} q_{k} q_{c}\left[(1-\alpha)-\theta s_{c}\right]^{2}-2 \tilde{\beta}^{\frac{1}{2}}\left(\tilde{\beta}^{\frac{1}{2}}-\cos \vartheta\right)\left[(1-\alpha)-\theta s_{c}\right] s_{k}^{-1} q_{c} q_{k} \\
& +q_{c}\left(q_{h}+q_{k}\right)\left[1+\tilde{\beta}-2 \tilde{\beta}^{\frac{1}{2}} \cos \vartheta\right]>0
\end{aligned}
$$


holds for all $-\pi \leq \vartheta \leq \pi$. Hence the second-order conditions are satisfied if and only if both $(i)$ and ( $i i)$ hold. (We can further reduce condition (ii) to a finite set of inequalities by considering the value of $\vartheta$ that minimizes the left-hand side expression.)

We note that when $q_{h}, q_{c}$ and $q_{k}$ are positive, as in the numerical examples considered in the text, then $(i)$ and $(i i)$ are always satisfied for all $-\pi \leq \vartheta \leq \pi$. The left-hand side of $(i i)$ can be equivalently written as

$$
\begin{aligned}
& \tilde{\beta} s_{k}^{-2} s_{c}^{2} q_{k} q_{h}+q_{c} q_{h}\left(\tilde{\beta}^{\frac{1}{2}}-\cos \vartheta\right)^{2}+q_{c}\left(q_{h}+q_{k}\right) \sin ^{2} \vartheta \\
& +q_{k} q_{c}\left[\tilde{\beta}^{\frac{1}{2}} s_{k}^{-1}\left((1-\alpha)-\theta s_{c}\right)-\left(\tilde{\beta}^{\frac{1}{2}}-\cos \vartheta\right)\right]^{2}
\end{aligned}
$$

and each of these terms is necessarily positive when $q_{h}, q_{c}$ and $q_{k}$ are positive.

\section{Euler Equation Residuals}

In the stochastic case, the exact Euler equations that characterize Ramsey optimal policy, corresponding to equations (1.32) and (1.34) of section 1.4, are given respectively by

$$
u_{c}\left(c_{t}, h_{t}\right)-\left[u_{c}\left(c_{t}, h_{t}\right)+u_{c c}\left(c_{t}, h_{t}\right) c_{t}+u_{h c}\left(c_{t}, h_{t}\right) h_{t}\right] \lambda_{0}-\lambda_{1, t}=0
$$

and

$$
\lambda_{1, t}-\beta E_{t}\left\{\left[f_{k}\left(k_{t+1}, z_{t+1} h_{t+1}\right)+(1-\delta)\right] \lambda_{1, t+1}\right\}=0,
$$

for each $t \geq t_{0}$. In terms of the detrended variables, these conditions can be rewritten as

$$
u_{c}\left(\tilde{c}_{t}, h_{t}\right)-\left[u_{c}\left(\tilde{c}_{t}, h_{t}\right)+u_{c c}\left(\tilde{c}_{t}, h_{t}\right) \tilde{c}_{t}+u_{h c}\left(\tilde{c}_{t}, h_{t}\right) h_{t}\right] \lambda_{0}-\tilde{\lambda}_{1, t}=0
$$

and

$$
\tilde{\lambda}_{1, t}-\tilde{\beta} E_{t}\left\{\left[\tilde{f}_{k}\left(\tilde{k}_{t+1}, \tilde{z}_{t+1} h_{t+1}\right)+(1-\tilde{\delta})\right] \tilde{\lambda}_{1, t+1}\right\}=0
$$

respectively, where

$$
\tilde{\lambda}_{1 t} \equiv \lambda_{1 t} \rho^{[1-\varphi(1-\gamma)] t},
$$

and the other detrended variables are defined as in section 1.3. ${ }^{35}$

\footnotetext{
${ }^{35}$ Note that equations (1.32) and (1.34) of section 1.4 are already written in terms of the detrended variables.
} 
Using (4.12) to eliminate $\tilde{\lambda}_{1 t}$ in (4.13), and again dropping the tildes from the detrended variables (that are henceforth to be understood), we obtain an Euler equation of the form

$$
\Lambda\left(c_{t}, h_{t}\right)=\tilde{\beta} E_{t}\left[\Lambda\left(c_{t+1}, h_{t+1}\right) r\left(k_{t+1} / z_{t+1} h_{t+1}\right)\right]
$$

where we define

$$
\begin{gathered}
\Lambda(c, h) \equiv u_{c}(c, h)-\lambda_{0}\left[u_{c}(c, h)+u_{c c}(c, h) c+u_{h c}(c, h) h\right] \\
r(\kappa) \equiv \tilde{f}_{k}(\kappa, 1)+(1-\tilde{\delta}) .
\end{gathered}
$$

This condition is equivalent to $R_{t}=0$, where we define the residual $R_{t}$ as the left-hand side of (4.14) minus the right-hand side, divided by $u_{c}(\bar{c}, \bar{h})$. The normalization implies that a value of .01 for the residual means that $\tilde{\lambda}_{1 t}$ exceeds $\tilde{\beta} E_{t}\left[\left(\tilde{f}_{k}+(1-\tilde{\delta})\right) \tilde{\lambda}_{1, t+1}\right]$ by an amount equal to one percent of the steady-state marginal utility of consumption.

The residual $R_{t}$ each period is given by a function $R\left(v_{t}\right)$ of the state vector $v_{t} \equiv$ $\left(\hat{k}_{t}, \hat{z}_{t}, \hat{g}_{t}\right)$ for that period of the simulation, where

$$
\begin{aligned}
R\left(v_{t}\right) & \equiv m\left(v_{t}\right)-E\left[n\left(v_{t+1}\right) \mid v_{t}\right] \\
& =m\left(v_{t}\right)-\sum_{s^{\prime}} \pi\left(s^{\prime} \mid s\left(v_{t}\right)\right) n\left(\hat{k}^{\prime}\left(v_{t}\right), s^{\prime}\right) .
\end{aligned}
$$

In this formula, $s\left(v_{t}\right)$ denotes the exogenous state $s_{t} \equiv\left(\hat{z}_{t}, \hat{g}_{t}\right)$ implied by the state vector $v_{t}$ (just the last two elements of the vector); $\pi\left(s^{\prime} \mid s\right)$ is the probability that the exogenous state $s_{t+1}=s^{\prime}$ given that $s_{t}=s$; the summation is over the possible values $s^{\prime}$ for the exogenous state $s_{t+1}$; and $\hat{k}^{\prime}\left(v_{t}\right)$ is the log-linear policy rule that gives the value of $\hat{k}_{t+1}$ as a function of $v_{t}$, in the solution to the LQ policy problem. ${ }^{36}$

Using the functional forms for preferences given in the text, the functions $m(v)$ and $n(v)$ are defined as

$$
\begin{gathered}
m(v) \equiv\left[1-\bar{\lambda}_{0} \varphi\left(1-\frac{\gamma}{1-\bar{h} \exp [\hat{h}]}\right)\right] \cdot \exp \left[(\varphi(1-\gamma) \hat{c}] \cdot\left(\frac{1-\bar{h} \exp [\hat{h}]}{1-\bar{h}}\right)^{\varphi \gamma},\right. \\
n(v) \equiv \tilde{\beta} m(v) r(v),
\end{gathered}
$$

\footnotetext{
${ }^{36}$ In computing the corresponding residuals in the case of the second-order perturbation solution, we define the function $R(v)$ in the same way, except that the function $\hat{k}^{\prime}(v)$ is now a quadratic approximation to the optimal policy rule.
} 
where

$$
r(v) \equiv\left[\tilde{\beta}^{-1}-(1-\tilde{\delta})\right] \cdot \exp [(1-\alpha)(\hat{z}+\hat{h}-\hat{k})]+(1-\tilde{\delta}) .
$$

In each of these last expressions, $\hat{c}, \hat{h}, \hat{z}$ and $\hat{k}$ should be understood to be functions of $v(\hat{c}(v)$, etc. $)$, in which the argument has been suppressed. The functions $\hat{z}(v)$ and $\hat{k}(v)$ select particular elements of the state vector $\left(\hat{z}_{t}\right.$ and $\hat{k}_{t}$ respectively), while the functions $\hat{c}(v)$ and $\hat{h}(v)$ represent the log-linear policy rules that specify the optimal values for $\hat{c}_{t}$ and $\hat{h}_{t}$ as functions of $v_{t} \cdot{ }^{37}$ Note that computation of the functions $m(v)$ and $n(v)$, and hence computation of the function $R(v)$, requires only numerical values for the model parameters $\varphi, \gamma, \tilde{\beta}, \tilde{\delta}$, and $\alpha$, and steady-state values for $\bar{h}$ and $\bar{\lambda}_{0}$.

\footnotetext{
${ }^{37}$ Again, in the case of the residuals for the second-order solution, the functions $\hat{c}(v)$ and $\hat{h}(v)$ are quadratic approximations to the optimal policy rules.
} 


\section{References}

[1] Benigno, Pierpaolo, and Michael Woodford (2004a), "Inflation Stabilization and Welfare: The Case of a Distorted Steady State," presented at the CEPR European Summer Symposium in Macroeconomics, Tarragona, Spain, May.

[2] Benigno, Pierpaolo, and Michael Woodford (2004b), "Linear-Quadratic Approximation of Optimal Policy Problems," in preparation.

[3] Bertsekas, Dimitri P. (1976), Dynamic Programming and Stochastic Control, New York: Academic Press.

[4] Chamley, Christophe (1986), "Optimal Taxation of Capital Income in General Equilibrium with Infinite Lives," Econometrica 54: pp. 607-622.

[5] Chari, V.V., Lawrence J. Christiano, and Patrick J. Kehoe (1994), "Optimal Fiscal Policy in a Business Cycle Model," The Journal of Political Economy vol. 102, No. 4, pp. 617-652.

[6] Chari, V.V., Lawrence J. Christiano, and Patrick J. Kehoe (1995), "Policy Analysis in Business Cycle Models," in T. F. Cooley (ed.), Frontiers of Business Cycle Research, Princeton: Princeton University Press.

[7] Chow, Gregory C. (1975), Analysis and Control of Dynamic Economic Systems John Wiley \& Sons, New York.

[8] Christiano, Lawrence J., and Martin Eichenbaum (1992), "Current RealBusiness-Cycle Theories and Aggregate Labor-Market Fluctuations,", American Economic Review, vol. 82, pp. 430-50.

[9] Hansen, Lars P., and Thomas J. Sargent (2004), Recursive Models of Dynamic Linear Economies, unpublished, University of Chicago, August 2004.

[10] Jin, Hehui, and Kenneth L. Judd (2002), "Perturbation Methods for General Dynamic Stochastic Models," unpublished, Stanford University.

[11] Judd, Kenneth L. (1985) "Redistributive Taxation in a Simple Perfect Foresight Model," Journal of Public Economics, vol. 28, pp. 59-83. 
[12] Judd, Kenneth L. (1996), "Approximation, Perturbation and Projection Methods in Economic Analysis," in H.M. Amman, J. Rust, and D.A. Kendrick (eds.), Handbook of Computational Economics, vol. I, Amsterdam: North Holland.

[13] Judd, Kenneth L. (1999), Numerical Methods In Economomics, Cambridge, Mass.: MIT Press.

[14] Kim, Jinill, and Dale W. Henderson, "Inflation Targeting and Nominal-IncomeGrowth Targeting: When and Why Are They Suboptimal?" Journal of Monetary Economics, forthcoming 2004.

[15] Kim, Jinill, and Kim, Henry S. (2003), "Spurious Welfare Reversal in International Business Cycle Models", Journal of International Economics, Volume 60, Issue 2, Pages 471-500 .

[16] Kim, Jinill, Sunghyun Kim, Ernst Schaumburg, and Christopher A. Sims (2003), "Calculating and Using Second Order Accurate Solutions of Discrete Time Dynamic Equilibrium Models," unpublished manuscript, University of Virginia, June.

[17] Kwakernaak, Huibert, and Raphael Sivan (1972), Linear Optimal Control Systems, New York: Wiley.

[18] Sargent, Thomas J. (1987), Macroeconomic Theory, 2d edition, New York: Academic Press.

[19] Schmitt-Grohé, Stephanie, and Martin Uribe (2004a), "Solving Dynamic General Equilibrium Models Using a Second-Order Approximation to the Policy Function," Journal of Economic Dynamics and Control, vol. 28, pp. 755-775.

[20] Schmitt-Grohé, Stephanie, and Martin Uribe (2004b), "Optimal Fiscal and Monetary Policy under Sticky Prices," Journal of Economic Theory vol. 114, pp. 198-230.

[21] Telser, Lester G., and Robert L. Graves (1972), Functional Analysis in Mathematical Economics: Optimization over Infinite Horizons, Chicago: University of Chicago Press. 
[22] Woodford, Michael (2002) "Inflation Stabilization and Welfare," Contributions to Macroeconomics 2, issue 1, article 1. [Online at www.bepress.com]

[23] Woodford, Michael (2003), Interest and Prices: Foundations of a Theory of Monetary Policy, Princeton: Princeton University Press. 
Table 1: Parameter values used in the numerical examples, following Chari et al. (1994).

\begin{tabular}{cc} 
& Calibrated Parameters \\
\hline$\alpha$ & \\
$\tilde{\beta}$ & 0.344 \\
$\gamma$ & 0.98 \\
$\tilde{\delta}$ & 0.75 \\
$\rho$ & 0.095 \\
$\bar{g}$ & 1.016 \\
& 0.069
\end{tabular}

Steady-State Values

$\begin{array}{lll}\varphi & 0 & -8 \\ \bar{\tau}^{h} & 0.2387 & 0.2069 \\ \bar{\tau}^{k} & 0 & 0 \\ s_{c} & .550 & .554 \\ s_{g} & .167 & .163 \\ s_{k} & 2.992 & 2.992 \\ \bar{h} & .232 & .238 \\ \bar{y} & .410 & .420 \\ \phi & .908 & .939\end{array}$

AR(1) Shock Processes

$$
\begin{array}{lllll}
\hat{z}_{t}: & \rho_{z}= & 0.81 & \sigma_{z}= & 0.041 \\
\hat{g}_{t}: & \rho_{g}= & 0.89 & \sigma_{g}= & 0.070
\end{array}
$$


Table 2: Coefficients of the quadratic loss function, for each of two alternative parameterizations.

\begin{tabular}{lll}
$\varphi$ & 0 & -8 \\
\hline & & \\
$q_{c}$ & 1 & 2.93 \\
$q_{h}$ & 0.45 & 1.23 \\
$q_{k}$ & 0.41 & 0.40 \\
$\theta$ & 0 & 0.79 \\
$\theta_{z}$ & 2.67 & 0.94
\end{tabular}


Table 3: Statistics on optimal tax rates according to the LQ approximation.

baseline high r.a. only z only g IID

Tax Rate on Labor Income

$\begin{array}{lrrrrr}\mathrm{E}(\tau) & 23.87 & 20.69 & 23.87 & 23.87 & 23.87 \\ \text { s.d. }(\tau) & .10 & .03 & .07 & .06 & .15 \\ \rho(\tau) & .77 & .81 & .69 & .90 & -.07 \\ \operatorname{corr}(\tau, \hat{g}) & .62 & -.55 & \mathrm{NA} & 1.00 & .10 \\ \operatorname{corr}(\tau, \hat{z}) & .49 & -.80 & .63 & \mathrm{NA} & .95\end{array}$

Ex Ante Tax Rate on Capital Income

$\begin{array}{lrrrrr}\mathrm{E}(\tau) & 0 & 0 & 0 & 0 & 0 \\ \text { s.d. }(\tau) & 0 & 3.29 & 0 & 0 & 0 \\ \rho(\tau) & \mathrm{NA} & .80 & \mathrm{NA} & \mathrm{NA} & \mathrm{NA} \\ \operatorname{corr}(\tau, \hat{g}) & \mathrm{NA} & .25 & \mathrm{NA} & \mathrm{NA} & \mathrm{NA} \\ \operatorname{corr}(\tau, \hat{z}) & \mathrm{NA} & .97 & \mathrm{NA} & \mathrm{NA} & \mathrm{NA}\end{array}$

Ex Post Tax Rate on Capital Income

$\begin{array}{lrrrrr}\mathrm{E}(\tau) & 0 & 0 & 0 & 0 & 0 \\ \text { s.d. }(\tau) & 36.13 & 30.56 & 15.77 & 32.51 & 10.81 \\ \rho(\tau) & 0 & -.00 & 0 & 0 & 0 \\ \operatorname{corr}(\tau, \hat{g}) & .41 & .44 & \mathrm{NA} & .46 & 0.91 \\ \operatorname{corr}(\tau, \hat{z}) & -.26 & -.13 & -.59 & \mathrm{NA} & -.41\end{array}$


Table 4: Statistics on optimal tax rates reported by Chari et al. (1994, Table 2). baseline high r.a. only z only g IID

Tax Rate on Labor Income

$\begin{array}{lrrrrr}\mathrm{E}(\tau) & 23.87 & 20.69 & 23.80 & 23.87 & 23.84 \\ \text { s.d. }(\tau) & .10 & .04 & .08 & .06 & .15 \\ \rho(\tau) & .80 & .85 & .71 & .90 & -.04 \\ \operatorname{corr}(\tau, \hat{g}) & .65 & -.59 & \mathrm{NA} & 1.00 & .10 \\ \operatorname{corr}(\tau, \hat{z}) & .55 & -.84 & .64 & \mathrm{NA} & .95\end{array}$

Ex Ante Tax Rate on Capital Income

$\begin{array}{lrrrrr}\mathrm{E}(\tau) & 0 & -.06 & 0 & 0 & 0 \\ \text { s.d. }(\tau) & 0 & 4.06 & 0 & 0 & 0 \\ \rho(\tau) & \mathrm{NA} & .83 & \mathrm{NA} & \mathrm{NA} & \mathrm{NA} \\ \operatorname{corr}(\tau, \hat{g}) & \mathrm{NA} & .33 & \mathrm{NA} & \mathrm{NA} & \mathrm{NA} \\ \operatorname{corr}(\tau, \hat{z}) & \mathrm{NA} & .96 & \mathrm{NA} & \mathrm{NA} & \mathrm{NA}\end{array}$

Ex Post Tax Rate on Capital Income

$\begin{array}{lrrrrr}\mathrm{E}(\tau) & .55 & -.42 & 1.19 & -.59 & .23 \\ \text { s.d. }(\tau) & 40.93 & 30.35 & 17.67 & 36.22 & 12.03 \\ \rho(\tau) & -.01 & .02 & .01 & .01 & -.02 \\ \operatorname{corr}(\tau, \hat{g}) & .40 & .47 & \mathrm{NA} & .46 & .94 \\ \operatorname{corr}(\tau, \hat{z}) & -.24 & -.02 & -.56 & \mathrm{NA} & .33^{*}\end{array}$

*We suspect this entry may be reported with a sign error. 


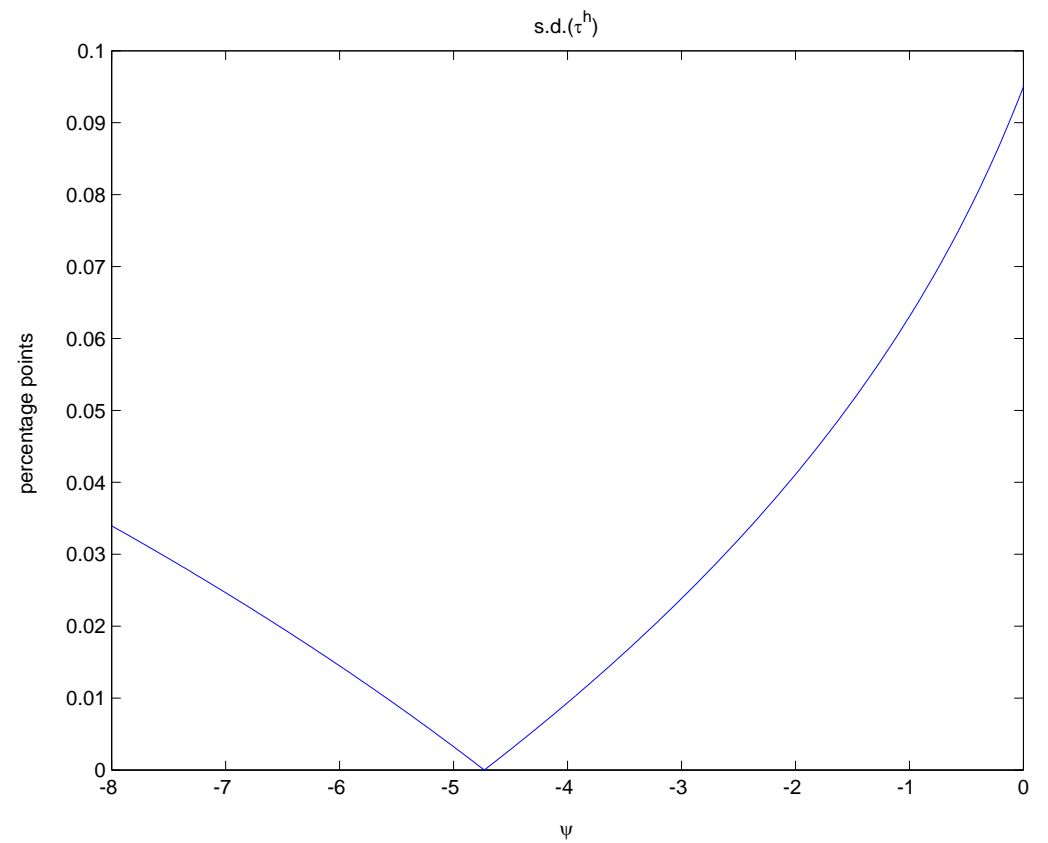

Figure 1: Variability of optimal tax rate on labor income for alternative values of $\varphi$, according to our LQ approximation. 


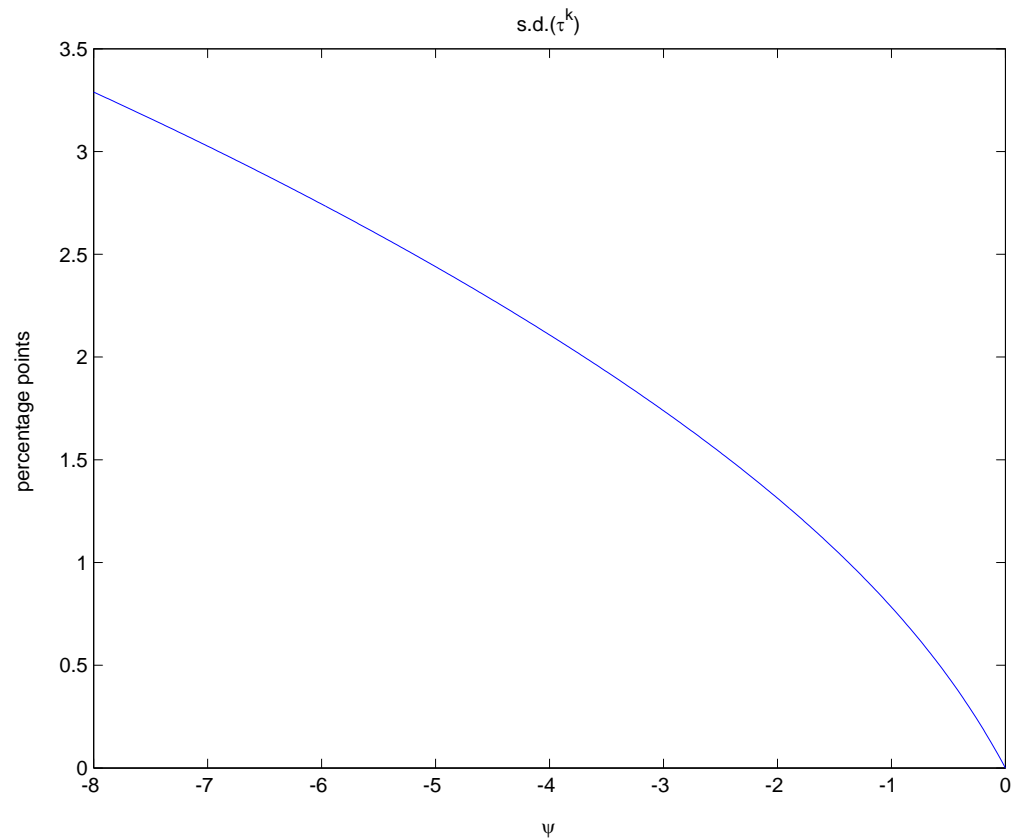

Figure 2: Variability of optimal ex ante tax rate on capital income for alternative values of $\varphi$, according to our LQ approximation. 

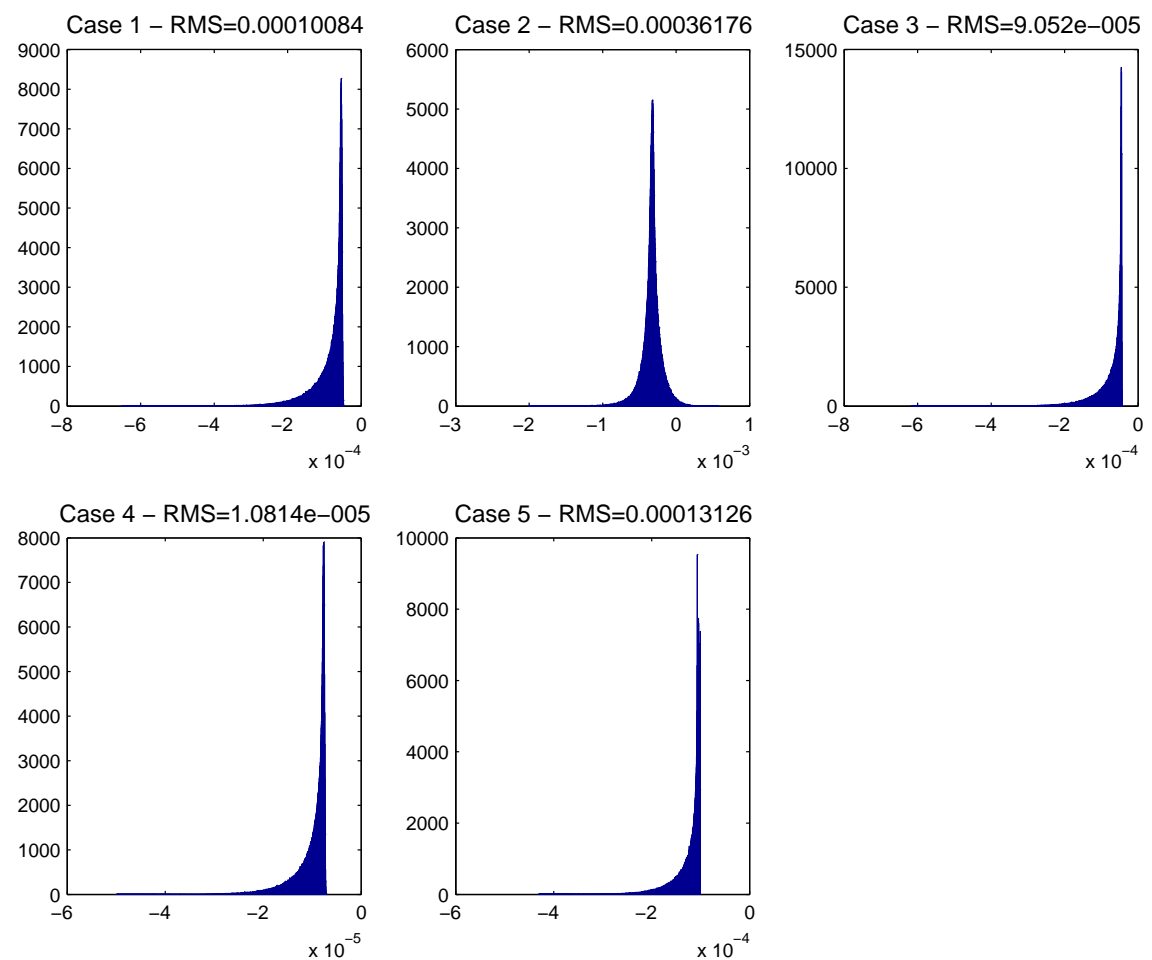

Figure 3: Distributions of Euler-equation residuals in the simulation of the log-linear dynamics that solve the LQ problem, for each of the 5 cases treated in Table 5 . The caption for each panel indicates the root-mean-square residual size in that simulation. 
Table 5: Statistics on optimal tax rates from Monte Carlo simulation of log-linearized optimal policy rules.

baseline high r.a. only z only g IID

Tax Rate on Labor Income

$\begin{array}{lrrrrr}\mathrm{E}(\tau) & 23.870 & 20.690 & 23.870 & 23.870 & 23.870 \\ \text { s.d. }(\tau) & .095 & .034 & .074 & .059 & .147 \\ \rho(\tau) & .766 & .811 & .685 & .895 & -.068 \\ \operatorname{corr}(\tau, \hat{g}) & .620 & -.550 & \mathrm{NA} & .999 & .099 \\ \operatorname{corr}(\tau, \hat{z}) & .496 & -.802 & .632 & \mathrm{NA} & .954\end{array}$

Ex Ante Tax Rate on Capital Income

$\begin{array}{lrrrrr}\mathrm{E}(\tau) & 0 & .002 & 0 & 0 & 0 \\ \text { s.d. }(\tau) & 0 & 3.289 & 0 & 0 & 0 \\ \rho(\tau) & \mathrm{NA} & .804 & \mathrm{NA} & \mathrm{NA} & \mathrm{NA} \\ \operatorname{corr}(\tau, \hat{g}) & \mathrm{NA} & .252 & \mathrm{NA} & \mathrm{NA} & \mathrm{NA} \\ \operatorname{corr}(\tau, \hat{z}) & \mathrm{NA} & .965 & \mathrm{NA} & \mathrm{NA} & \mathrm{NA}\end{array}$

Ex Post Tax Rate on Capital Income

$\begin{array}{lrrrrr}\mathrm{E}(\tau) & .001 & .003 & -.003 & .004 & .001 \\ \text { s.d. }(\tau) & 36.155 & 30.581 & 15.769 & 32.512 & 10.818 \\ \rho(\tau) & -.000 & -.003 & -.002 & -.000 & -.000 \\ \operatorname{corr}(\tau, \hat{g}) & .410 & .444 & \mathrm{NA} & .456 & 0.913 \\ \operatorname{corr}(\tau, \hat{z}) & -.255 & -.132 & -.586 & \mathrm{NA} & -.409\end{array}$


Table 6: Statistics on optimal tax rates from a second-order approximation to the policy rules.

baseline high r.a. only z only g IID

Tax Rate on Labor Income

$\begin{array}{lrrrrr}\mathrm{E}(\tau) & 23.873 & 20.687 & 23.870 & 23.873 & 23.871 \\ \text { s.d. }(\tau) & .095 & .034 & .074 & .059 & .147 \\ \rho(\tau) & .766 & .811 & .685 & .895 & -.068 \\ \operatorname{corr}(\tau, \hat{g}) & .621 & -.550 & \mathrm{NA} & 1.000 & .099 \\ \operatorname{corr}(\tau, \hat{z}) & .496 & -.802 & .632 & \mathrm{NA} & .954\end{array}$

Ex Ante Tax Rate on Capital Income

$\begin{array}{lrrrrr}\mathrm{E}(\tau) & 0 & .027 & 0 & 0 & 0 \\ \text { s.d. }(\tau) & 0 & 3.316 & 0 & 0 & 0 \\ \rho(\tau) & \mathrm{NA} & .803 & \mathrm{NA} & \mathrm{NA} & \mathrm{NA} \\ \operatorname{corr}(\tau, \hat{g}) & \mathrm{NA} & .250 & \mathrm{NA} & \mathrm{NA} & \mathrm{NA} \\ \operatorname{corr}(\tau, \hat{z}) & \mathrm{NA} & .957 & \mathrm{NA} & \mathrm{NA} & \mathrm{NA}\end{array}$

Ex Post Tax Rate on Capital Income

$\begin{array}{lrrrrr}\mathrm{E}(\tau) & .529 & .030 & .953 & -.435 & .503 \\ \text { s.d. }(\tau) & 36.302 & 30.770 & 15.801 & 32.513 & 10.914 \\ \rho(\tau) & -.001 & -.004 & -.002 & -.000 & -.000 \\ \operatorname{corr}(\tau, \hat{g}) & .409 & .442 & \mathrm{NA} & .456 & 0.905 \\ \operatorname{corr}(\tau, \hat{z}) & -.254 & -.132 & -.585 & \mathrm{NA} & -.406\end{array}$

